\title{
RELATIVISTIC LINES AND REFLECTION FROM THE INNER ACCRETION DISKS AROUND NEUTRON STARS
}

\author{
Edward M. Cackett ${ }^{1,8}$, Jon M. Miller ${ }^{1}$, David R. Ballantyne ${ }^{2}$, Didier Barret ${ }^{3}$, Sudip Bhattacharyya $^{4}$, \\ Martin Boutelier $^{3}$, M. Coleman Miller ${ }^{5}$, Tod E. Strohmayer ${ }^{6}$, and Rudy Wijnands ${ }^{7}$ \\ ${ }^{1}$ Department of Astronomy, University of Michigan, Ann Arbor, MI 48109, USA; ecackett@ umich.edu \\ ${ }^{2}$ Center for Relativistic Astrophysics, School of Physics, Georgia Institute of Technology, Atlanta, GA 30332, USA \\ ${ }^{3}$ Centre d'Etude Spatiale des Rayonnements, CNRS/UPS, 9 Avenue du Colonel Roche, 31028 Toulouse Cedex04, France \\ ${ }^{4}$ Department of Astronomy and Astrophysics, Tata Institute of Fundamental Research, Mumbai 400005, India \\ ${ }^{5}$ Department of Astronomy, University of Maryland, College Park, MD 20742-2421, USA \\ ${ }^{6}$ Astrophysics Science Division, NASA/GSFC, Greenbelt, MD 20771, USA \\ ${ }^{7}$ Astronomical Institute “Anton Pannekoek," University of Amsterdam, Science Park 904, 1098 XH, Amsterdam, The Netherlands \\ Received 2009 August 9; accepted 2010 July 4; published 2010 August 6
}

\begin{abstract}
A number of neutron star low-mass X-ray binaries (LMXBs) have recently been discovered to show broad, asymmetric $\mathrm{Fe} \mathrm{K}$ emission lines in their X-ray spectra. These lines are generally thought to be the most prominent part of a reflection spectrum, originating in the inner part of the accretion disk where strong relativistic effects can broaden emission lines. We present a comprehensive, systematic analysis of Suzaku and XMM-Newton spectra of 10 neutron star LMXBs, all of which display broad Fe K emission lines. Of the 10 sources, 4 are $\mathrm{Z}$ sources, 4 are atolls, and 2 are accreting millisecond X-ray pulsars (also atolls). The $\mathrm{Fe} \mathrm{K}$ lines are fit well by a relativistic line model for a Schwarzschild metric, and imply a narrow range of inner disk radii (6-15 $\left.G M / c^{2}\right)$ in most cases. This implies that the accretion disk extends close to the neutron star surface over a range of luminosities. Continuum modeling shows that for the majority of observations, a blackbody component (plausibly associated with the boundary layer) dominates the X-ray emission from 8 to $20 \mathrm{keV}$. Thus it appears likely that this spectral component produces the majority of the ionizing flux that illuminates the accretion disk. Therefore, we also fit the spectra with a blurred reflection model, wherein a blackbody component illuminates the disk. This model fits well in most cases, supporting the idea that the boundary layer illuminates a geometrically thin disk.
\end{abstract}

Key words: accretion, accretion disks - stars: neutron - X-rays: binaries

\section{INTRODUCTION}

X-ray emission lines from the innermost accretion disks are well known in supermassive and stellar-mass black holes, where they are shaped by relativistic effects (for a review, see Miller 2007). The utility of such lines is two-fold: they can be used to constrain the spin of the black hole, and they can be used to constrain the nature of the innermost accretion flow, particularly the proximity of the disk to the black hole (Brenneman \& Reynolds 2006; Miniutti et al. 2009; Reis et al. 2009a; Miller et al. 2006a, 2008b, 2009; Reynolds et al. 2009; Wilkinson \& Uttley 2009; Schmoll et al. 2009; Fabian et al. 2009).

Disk lines are produced in a fairly simple manner: a source of hard X-ray emission that is external to the disk is all that is required. The specific nature of the hard X-ray emission - whether thermal or non-thermal, whether due to Compton upscattering (e.g., Gierliński et al. 1999) or emission from the base of a jet (Markoff \& Nowak 2004; Markoff et al. 2005), or even a hot blackbody-is less important than the simple fact of a continuum source with substantial ionizing flux. The most prominent line produced in this process is typically an $\mathrm{Fe} \mathrm{K}$ line, due to its abundance and fluorescent yield; however, other lines can also be produced. The overall interaction is known as "disk reflection" and more subtle spectral features, including a "reflection hump" peaking between 20 and $30 \mathrm{keV}$, are also expected (see, e.g., George \& Fabian 1991; Magdziarz \& Zdziarski 1995; Nayakshin \& Kallman 2001; Ballantyne et al. 2001; Ross \& Fabian 2007).

\footnotetext{
8 Chandra Fellow.
}

Largely owing to a combination of improved instrumentation and concerted observational efforts within the last two years, asymmetric $\mathrm{Fe} \mathrm{K}$ disk lines have been observed in eight neutron star low-mass X-ray binaries (LMXBs; Bhattacharyya \& Strohmayer 2007; Cackett et al. 2008; Pandel et al. 2008; D'Aì et al. 2009; Cackett et al. 2009a; Papitto et al. 2009; Shaposhnikov et al. 2009; Reis et al. 2009b; Di Salvo et al. 2009c; Iaria et al. 2009). In the case of neutron stars, disk lines can be used in much the same way as in black holes to determine the inner disk radius. The radius of a neutron star is critical to understanding its equation of state; disk lines set an upper limit since the stellar surface (if not also a boundary layer) truncates the disk. In the case of neutron stars harboring pulsars, disk lines can be used to trace the radial extent of the disk and to obtain a magnetic field constraint (Cackett et al. 2009a). Finally, in a number of neutron stars, hot quasi-blackbody emission (potentially from the boundary layer) provides most of the flux required to ionize iron; the fact that the disk intercepts this flux suggests that the inner disk is geometrically thin, or at least thinner than the vertical extent of the boundary layer.

Disk line spectroscopy provides an independent view on the inner accretion flow in neutron star LMXBs, wherein some constraints have been derived using X-ray timing (for a review, see van der Klis 2006). The higher frequency part of so-called $\mathrm{kHz}$ quasi-periodic oscillation (QPO) pairings may reflect the Keplerian orbital frequency in the inner disk (e.g., Stella \& Vietri 1998), for instance. Trends in the coherence of the lowfrequency part of $\mathrm{kHz}$ QPO pairs may indicate the point at which the disk has reached an inner stable orbit (Barret et al. 2006), though see Méndez (2006) for an opposing view. Thus, 
Table 1

Observation Details

\begin{tabular}{|c|c|c|c|c|c|c|c|c|c|}
\hline Source & Class & Mission & Obs. ID & Obs. Start Date (dd/mm/yyyy) & Exp. Time (ks) & Mode & Ref. & Distance $(\mathrm{kpc})$ & Distance Ref. \\
\hline \multirow[t]{2}{*}{ Serpens X-1 } & A & Suzaku & 401048010 & $24 / 10 / 2006$ & $18 / 29$ & $1 / 4 \mathrm{~W}, 1.0 \mathrm{~s}$ & 1 & 8.4 & 11 \\
\hline & & $X M M-N e w t o n$ & 0084020501 & $24 / 03 / 2004$ & 7 & PN: timing & 2 & & \\
\hline \multirow[t]{3}{*}{$4 \mathrm{U} 1636-53$} & A & $X M M-N e w t o n$ & 0303250201 & $29 / 08 / 2005$ & 29 & PN: timing & 3 & $6.0 \pm 0.1$ & 12 \\
\hline & & $X M M-N e w t o n$ & 0500350301 & $28 / 09 / 2007$ & 26 & PN: timing & 3 & & \\
\hline & & XMM-Newton & 0500350401 & $27 / 02 / 2008$ & 37 & PN: timing & 3 & & \\
\hline & & Suzaku & 401046030 & $06 / 10 / 2006$ & $18 / 17$ & $1 / 4 \mathrm{~W}, 2.0 \mathrm{~s}$ & 4 & & \\
\hline & & XMM-Newton & 0402300201 & $26 / 08 / 2006$ & 34 & PN: timing & 5 & & \\
\hline $4 \mathrm{U} 1820-30$ & A & Suzaku & 401047010 & $14 / 09 / 2006$ & $10 / 30$ & $1 / 4 \mathrm{~W}, 1.0 \mathrm{~s}$ & 1 & $7.6 \pm 0.4$ & 13 \\
\hline \multirow[t]{2}{*}{ GX 17+2 } & Z & Suzaku & 402050010 & $19 / 09 / 2007$ & $5 / 15$ & $1 / 4 \mathrm{~W}, 0.5 \mathrm{~s}$ & 5 & $9.8 \pm 0.4$ & 12 \\
\hline & & Suzaku & 402050020 & $27 / 09 / 2007$ & $6 / 18$ & $1 / 4 \mathrm{~W}, 0.5 \mathrm{~s}$ & 5 & & \\
\hline GX $340+0$ & Z & $X M M-N e w t o n$ & 0505950101 & $02 / 09 / 2007$ & 33 & PN: timing & 6 & $11 \pm 3$ & 14 \\
\hline \multirow[t]{2}{*}{ SAX J1808.4-3658 } & AMXP & Suzaku & 903003010 & $02 / 10 / 2008$ & $21 / 31$ & $1 / 4 \mathrm{~W}, 1.0 \mathrm{~s}$ & 9 & $3.5 \pm 0.1$ & 15 \\
\hline & & XMM-Newton & 0560180601 & $30 / 09 / 2008$ & 43 & PN: timing & 9,10 & & \\
\hline HETE J1900.1-2455 & AMXP & Suzaku & 402016010 & $16 / 10 / 2007$ & $42 / 37$ & $1 / 4 \mathrm{~W}, 2.0 \mathrm{~s}$ & 5 & $3.6 \pm 0.5$ & 12 \\
\hline
\end{tabular}

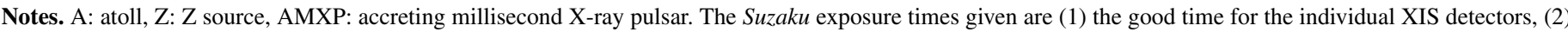

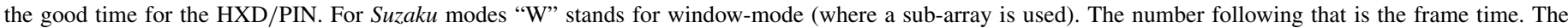

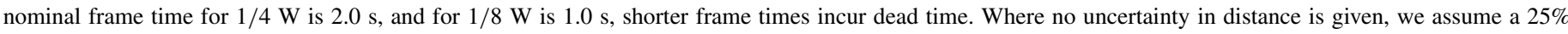
uncertainty.

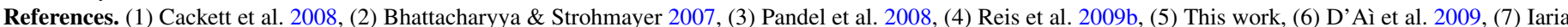

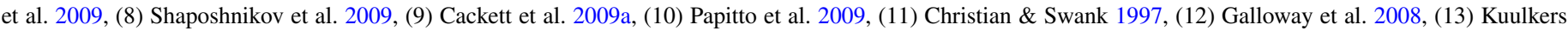
et al. 2003, (14) Fender \& Hendry 2000, (15) Galloway \& Cumming 2006.

X-ray timing and spectroscopy offer independent windows on the neutron star and inner accretion flow in LMXBs. It has been noted that with the assumption of Keplerian frequencies, combining the upper $\mathrm{kHz}$ QPO frequency with the radius given by a line measurement yields a mass determination (Piraino et al. 2000; Cackett et al. 2008).

In order to understand possible differences in the innermost accretion flow onto neutron star LMXBs, and in order to derive joint constraints on fundamental neutron star parameters, it is important to analyze the full sample of disk lines using a consistent, systematic approach. Herein, we analyze archival data from Suzaku (Mitsuda et al. 2007) and XMM-Newton (Jansen et al. 2001), using consistent and systematic reduction and modeling procedures. Each spectrum is fit both with phenomenological models and with physically motivated disk reflection models. In addition to $\mathrm{Fe} \mathrm{K}$ lines reported previously, we also present the discovery of broad lines in two additional sources, GX 17+2 and HETE J1900.1-2455. The total number of neutron star LMXBs with relativistic lines now stands at 10.

\section{SAMPLE OF NEUTRON STARS}

In this paper, we analyze data from 10 neutron star LMXBs, 8 of which have recently had detections of broad Fe K emission lines (Bhattacharyya \& Strohmayer 2007; Cackett et al. 2008; Pandel et al. 2008; D'Aì et al. 2009; Cackett et al. 2009a; Papitto et al. 2009; Shaposhnikov et al. 2009; Reis et al. 2009b; Di Salvo et al. 2009c; Iaria et al. 2009). In addition, we also present evidence for relativistic $\mathrm{Fe} \mathrm{K}$ emission lines in 2 further sources (GX 17+2 and HETE J1900.1-2455). The total sample consists of a variety of different types of neutron star LMXBs: 4 atolls (Ser X-1, 4U 1636-53, 4U 1705-44, 4U 1820-30), $4 \mathrm{Z}$ sources (GX 17+2, GX 340+0, GX 349+2, Cyg X-2), and 2 accreting millisecond X-ray pulsars (SAX J1808.4-3658 and HETE J1900.1-2455, which are also atolls). The observations we analyze here were obtained with either Suzaku or XMM-Newton, both of which have a good effective area through the Fe K region (6.4-6.97 keV). In Table 1, we detail the observations presented here, as well as give the distance to each source assumed throughout the paper.

\section{DATA REDUCTION}

Details of our data reduction for the Suzaku and XMM-Newton observations follow. We have attempted to follow a standard, common analysis procedure for all sources as much as possible. Where we have had to deviate from this, specific details for those observations are also given.

\subsection{Suzaku Data Reduction}

The data reduction was performed using HEASOFT v6.6.2, and the latest calibration files (as of 2009 June). Suzaku consists of both a set of soft X-ray CCD detectors (XIS) as well as a separate hard X-ray detector (HXD). Details of the data reduction for the XIS and HXD are given below. For all sources we adopted the following standard data reduction method, except where explicitly stated. Since our original analysis of Ser X-1, GX 349+2, and 4U 1820-30 (Cackett et al. 2008), there has been significant advances in the analysis tools for Suzaku, for instance, one can now generate response files for any source region (allowing for pile-up correction). 


\subsubsection{XIS Data Reduction}

First, we reprocessed the unfiltered event files using xispi, which updates the pulse-invariant values for the latest calibration. From there, we then created a cleaned, filtered event list using the standard screening criteria provided by Suzaku. In addition to the standard screening we were also careful in filtering out any periods where the telemetry was saturated. This only occurred in a very small number of cases, which are noted below. There are event lists for each data mode used on each detector. We treated all of them separately until after spectra and responses were generated.

There are a couple of exceptions to this procedure. When a source is bright, the script to remove flickering pixels (cleansis) sometimes produces spurious results, rejecting all the brightest pixels in the image. This was the case for a portion of both the second Cyg X-2 observation and the Ser X-1 observation. To overcome this, one must run cleansis without any iterations (as recommended in the cleansis user guide). We adopted this procedure for the few observations where this occurs.

From the cleaned, filtered event files we extracted spectra. Source extraction regions were chosen to be a rectangular box of $250 \times 400$ pixels for $1 / 4$ window mode observations and $125 \times 400$ pixels for $1 / 8$ window mode observations. For the brightest sources where pile-up is present, we excluded a circular region centered on the source, the radius of which depended on the severity of pile-up (radii are given below).

After the extraction of spectra, we generated $\mathrm{rmf}$ and arf files using xisrmfen and xissimarfgen (using 200,000 simulated photons) for each spectrum. We combined all the spectra and responses for the separate data modes (e.g., $2 \times 2,3 \times 3$ ) used for each detector using the addascaspec tool. Additionally, we then combined the spectra from all available front-illuminated detectors (XIS 0,2, and 3). The spectra were then rebinned by a factor of 4 to more closely match the HWHM of the spectral resolution.

We now give observation-specific details for each source.

Ser $X-1$. A central circular region of radius 30 pixels was excluded from the extraction region. There is also a small amount of telemetry saturation. We exclude times when this occurs, which corresponds to removing $3 \%$ of XIS 0,2 , and 3 events, and $0.1 \%$ of XIS 1 events.

$4 U$ 1705-44. In the first and third Suzaku observations no pile-up correction was needed. However, in the second observation, when the source was significantly brighter, a central circular region of radius 20 pixels was excluded. In the first observation, two type-I X-ray bursts were seen in the light curve, which we filtered out. No bursts were detected in the other two observations.

$4 U$ 1820-30. A central circular region of radius 40 pixels was excluded from the extraction region. There is also some telemetry saturation, and we exclude times when this occurs. This removes approximately $16 \%$ of events on all XIS detectors.

$G X 17+2$. A central circular region of radius 30 pixels was excluded from the extraction region for both observations of this source. Only XIS 0 and XIS 3 data are analyzed-XIS 2 was not in operation, and XIS 1 was not operated in window mode. We detected two X-ray bursts (one during each observation), which were excluded.

$G X 349+2$. A central circular region of radius 30 pixels was excluded from the extraction region for both observations of this source. The XIS 0,2 , and 3 detectors were operated in $1 / 8$ window mode, with a $0.3 \mathrm{~s}$ integration time. The XIS 1 detector, however, was operated in $1 / 4$ window mode, but also with a $0.3 \mathrm{~s}$ integration time, and thus has a smaller live time fraction (15\% compared to $30 \%$ ).

Cyg $X$-2. The first observation of this source has previously been published by Shaposhnikov et al. (2009). These authors noted that a large fraction of this observation was affected by telemetry saturation and thus only used data from times when high telemetry rates were utilized. On re-analysis, we found that even during high telemetry rates the data still suffered telemetry saturation. Strictly filtering the data using time where there was no telemetry saturation leads to less than $500 \mathrm{~s}$ of good time for each detector. Due to the resulting low count statistics, we do not analyze this observation further.

During the second observation, Cyg X-2 happened to be observed in a particularly bright phase, and we therefore excluded a central circular region of radius 75 pixels (see the later discussion of pile-up in Section 4.1.1). We only extract spectra from XIS 1 and XIS 3. XIS 0 was operated in "PSUM" (timing) mode. This mode is not yet calibrated; thus the spectra cannot be used for analysis. There was significant telemetry saturation during this observation, and we remove all times when this occurs. This leads to the exclusion of $36 \%$ of events from XIS 1 and 9\% from XIS 3.

SAX J1808.4-3658. We use the spectra from Cackett et al. (2009a), and do not reprocess the data here.

HETE J1900.1-2455. We only use sections of the data that were operated in $1 / 4$ window mode. Given the low source count rate, no pile-up correction was required.

\subsubsection{HXD/PIN Data Reduction}

In every case we downloaded the latest "tuned" background file from the Suzaku Web site. The PIN spectrum was extracted from the cleaned event file using good time intervals common to both the PIN event file and the "tuned" background file. The resulting source spectrum was then dead-time corrected. The non-X-ray background spectrum was extracted from the "tuned" background file, using the same common good time intervals. In addition to the non-X-ray background, the cosmic $X$-ray background also contributes a small $(\sim 5 \%)$ amount to the total background. We fake a cosmic X-ray background spectrum following the standard Suzaku procedure, and add this to the non-X-ray background spectrum to create the total background spectrum. The response of the detector has changed over time; thus, we make sure that we used the correct response associated with the epoch of the observation. In the few cases where type-I $\mathrm{X}$-ray bursts were detected in the XIS light curves, we also filtered out the same times from the HXD/PIN data.

\subsection{XMM-Newton Data Reduction}

We processed the observation data files (ODFs) for each observation using the XMM-Newton Science Analysis Software (v8). For the analysis here we only use timing mode data from the PN camera as it is the appropriate observing mode for the sources of interest. Calibrated event lists were created from the ODFs using the PN processing tool epproc. Exceptions to this procedure are the observations of SAX J1808.4-3658 and GX $340+0$. We obtained the calibrated event lists for these observations directly from the XMM-Newton Science Operations Center.

Before extracting spectra, we checked for periods of high background by extracting a light curve from an off-source strip with energy $>10 \mathrm{keV}$. We note where we found high 
background levels below. Using only times of low background, we extracted spectra with the following criteria: quality flag $=0$, pattern $\leqslant 4$ (singles and doubles) and in the energy range $0.3-12 \mathrm{keV}$. The source extraction regions used were 24 pixels wide (in RAWX), covered all RAWY values and were centered on the brightest RAWX column. In a few cases, where the source was particularly bright, we had to exclude the brightest two RAWX columns (details noted below). The corresponding rmf and arf were created using the rmf gen and arfgen tools following the Scientific Analysis System (SAS) procedures.

Observation-specific details follow.

Ser $X-1$. During the first observation, there is only a slightly high background at the beginning, which we choose to include. There is no background flaring in the other two observations.

$4 U$ 1636-53. There were three X-ray bursts during the first observation, one during the second observation, and one during the third observation, all of which were excluded. Only during the second observation there was some background flaring. Removing this reduced the exposure time by $3.5 \mathrm{ks}$.

$4 U$ 1705-44. One type-I X-ray burst occurred during this observation, and was excluded. We find no background flaring. Note that this is not the data set presented in Di Salvo et al. (2009c), which is still proprietary at the time of writing. Other archival XMM-Newton observations of this source were observed in imaging mode, and so are not analyzed here.

$G X 340+0$. Some low-level background flaring that reduces the exposure time by $7 \mathrm{ks}$. The source is particularly bright during this observation (see light curve in D'Aì et al. 2009); we therefore remove the two brightest columns from the extract region. Given that D'Aì et al. (2009) demonstrate that the Fe $\mathrm{K}$ line properties do not vary significantly with source state, we chose not to split the observation based on source state, but instead prefer to analyze the entire time-averaged spectrum.

$G X 349+2$. The source count rate is high during this observation (Iaria et al. 2009). Following Iaria et al. (2009), we therefore exclude the two brightest columns from the extraction region. There is no significant background flaring. Another archival observation of this source is also available, however, it was observed in imaging mode, and thus is not analyzed here.

SAX J1808.4-3658. We use the spectra from Cackett et al. (2009a) and do not reprocess the data here.

\section{SPECTRAL FITTING}

\subsection{Phenomenological Models}

Our first step is to fit the spectra with phenomenological models (throughout the paper we use XSPEC v12; Arnaud 1996). There has been debate for many years as to which is the most appropriate continuum model to fit to neutron star LMXBs (e.g., White et al. 1988; Mitsuda et al. 1989), as it is often the case that several different models fit the data equally well (e.g., Barret 2001). A recent investigation by Lin et al. (2007) studied two atoll sources over a wide range in luminosity. They investigated the luminosity dependence of measured temperatures for different continuum models, looking to see for which model choice the measured temperatures followed $L \propto T^{4}$. Based on this, they suggested a prescription for spectral fitting, using an absorbed disk blackbody (xspec model: diskbb; Arnaud 1996) plus single temperature blackbody plus a (broken) power law for the soft and intermediate states, and a blackbody plus broken power law for the hardest states. We have also found this model to fit the spectra of neutron star LMXBs well (Cackett et al. 2008, 2009a, 2009b). Here, we adopt this same model (though we use a power law, rather than a broken power law), testing that the addition of each component statistically improves the fit.

We interpret the hot blackbody component as the boundary layer between the accretion disk and neutron star surface (e.g., Inogamov \& Sunyaev 1999; Popham \& Sunyaev 2001). One can also successfully model this component with a Comptonized model, such as comptt. When doing this, however, one gets high optical depths - the Comptonized component is close to a Wien spectrum. Rather than fitting the more complicated Comptonization model, we chose the simpler blackbody model.

The millisecond X-ray pulsar, HETE J1900.1-2455, has a much harder spectrum than the other sources we study here. In fact, we find that its broadband continuum spectrum can be well fit by a single power law only.

We found that several of the sources have an apparent emission feature at around $1 \mathrm{keV}$, as has previously been observed in a number of neutron star LMXBs, with a number of different missions (e.g., Vrtilek et al. 1988; Kuulkers et al. 1997; Schulz 1999). It has suggested that this feature is a blend of a number of emission lines from $\mathrm{Fe}$ and $\mathrm{O}$ (Vrtilek et al. 1988). Here, if the feature is present, we fit with a single Gaussian emission line.

The presence of a broad $\mathrm{Fe} \mathrm{K}$ emission line in eight of these sources has already been established elsewhere (see references earlier). Of the other two sources (GX 17+2 and HETE J1900.1 - 2455) a Gaussian Fe K emission has previously been reported in GX 17+2 (Di Salvo et al. 2000b; Farinelli et al. 2005; Cackett et al. 2009b) and the fact that a broad red wing has not been previously observed is likely due to sensitivity (Cackett et al. 2009b). Here we show that the profile is asymmetric. In HETE J1900.1-2455, we detect a broad Fe K emission line for the first time, though the line can be fit equally well by a broad Gaussian or a relativistic line due to the low signal-tonoise ratio. Therefore, all 10 objects studied here are seen to have broad Fe $\mathrm{K}$ lines.

To fit these emission lines, we use the relativistic line model for a Schwarzschild metric (diskline; Fabian et al. 1989). The Schwarzschild metric should be a good approximation for neutron stars-for neutron stars with reasonable equations of state and spin frequencies less than $600 \mathrm{~Hz}$, the dimensionless angular momentum parameter is expected to be less than 0.3; thus any deviations from the Schwarzschild metric are minor (see Miller et al. 1998 for further discussion of this). We note that the diskline profile does not account for light bending, and so does not fully account for all relativistic effects. However, it still remains the most appropriate available model here.

We include this model in addition to the continuum model discussed above. The emission-line parameters are the line energy, disk emissivity index, inner disk radius, outer disk radius, and inclination. We constrain the line energy to be within 6.4-6.97 keV, the allowed range for $\mathrm{Fe} \mathrm{K} \alpha$ emission. The disk emissivity index and inner disk radius are left as free parameters in the fits. In most cases, the inclination is left as a free parameter (we discuss the exceptions to this later). Finally, we fix the outer disk radius at $1000 \mathrm{GM} / \mathrm{c}^{2}$. Typical disk emissivities mean that the $\mathrm{Fe} \mathrm{K}$ emission is quite centrally concentrated, and hence the outer disk radius is usually not constrained well and has little effect on the measured inner disk radius.

Several of the sources considered here have multiple observations by the same, or different telescopes. The inclination of the source should not change over time; thus, we should expect to recover the same inclination from the Fe $\mathrm{K}$ line modeling 
for each observation. In order to investigate the extent to which this is true, we chose to let the inclination parameter vary from observation to observation.

For the Suzaku spectra, we fit the XIS spectra over the energy range $1.0-10 \mathrm{keV}$, ignoring $1.5-2.5 \mathrm{keV}$ where large residuals are always seen (regardless of the count rate), presumably of an instrumental nature. The PIN spectra are fit from $12 \mathrm{keV}$ upward, with the upper energy depending on where the background dominates over the source spectrum. The combined frontilluminated spectrum, XIS 1 spectrum, and HXD/PIN spectra are all fit simultaneously, with a floating constant used to account for any absolute flux calibration offset between them. When fitting the XMM-Newton PN spectra, we fit over the $0.8-10 \mathrm{keV}$ range. Source specific details including exceptions to this standard procedure follow.

Ser X-1. Suzaku/PIN spectrum fit from 12 to $25 \mathrm{keV}$. In the $X M M-N e w t o n$ spectra, there are significant residuals around $1 \mathrm{keV}$ that are fit well by a Gaussian emission line.

$4 U$ 1636-53. The Fe line profile observed in this source (Pandel et al. 2008) is unlike what is seen in any of the other sources. It has a blue wing that extends to high energies, suggestive of a high inclination. Our spectral fitting does give a best fit with a high inclination (we find that all cases peg at $90^{\circ}$ ) and Pandel et al. (2008) also reported high inclinations (greater than $64^{\circ}$ in all cases). However, no eclipses have been detected in this source and optical measurements are not especially constraining $\left(36^{\circ}-74^{\circ}\right.$; Casares et al. 2006).

$4 U$ 1705-44. Suzaku/PIN spectrum fit from 12 to $30 \mathrm{keV}$. The first Suzaku observation has quite low sensitivity through the Fe K band (see Reis et al. 2009b). We therefore fix several of the line parameters.

When fitting the XMM-Newton data of 4U 1705-44, we fit in the range $1.2-10 \mathrm{keV}$, as a relatively high $N_{\mathrm{H}}$ and low source flux lead to low statistics below $1.2 \mathrm{keV}$. We found that both the diskbb + bbody and the bbody + power-law model fit the continuum spectra well, with the diskbb + bbody model having a marginally better $\chi^{2}$ value. The spectrum is of fairly low quality through the Fe $\mathrm{K}$ region, and thus a simple Gaussian models the Fe K line well. However, to allow comparison with other observations we still fit the line with the diskline model. When including the diskline model both choices of the continuum model give consistent $\mathrm{Fe} \mathrm{K}$ parameters, with almost identical $\chi^{2}$ values: 1762.4 versus 1762.5 (dof $=1752$ in both cases) for diskbb + bbody and bbody + power-law continuum choices, respectively.

An unphysically high inclination is determined from the $X M M-N e w t o n$ observation. This source is not known to be eclipsing or dipping. There is a different inclination determined in the Suzaku observations (Reis et al. 2009b) than from Di Salvo et al. (2009c). We revisit this issue later when discussing systematic uncertainties in Fe K modeling.

$4 U$ 1820-30. Suzaku/PIN spectrum fit from 12 to $25 \mathrm{keV}$. With the improved calibration, the combined front-illuminated spectrum has a significantly higher signal-to-noise ratio than the spectra presented by Cackett et al. (2008). With this improved spectrum, the detected emission line is found to be weaker than before, but still a significant detection: with the emission line the best fit has $\chi^{2}=1269$ for 1108 degrees of freedom (dof), whereas with no emission line the best fit has $\chi^{2}=1362$ for 1113 dof.

GX 17+2. Suzaku/PIN spectrum fit from 12 to $40 \mathrm{keV}$.

$G X 340+0$. This source is hampered by a very large column density $\left(N_{\mathrm{H}} \sim 10^{23} \mathrm{~cm}^{-2}\right)$. This means that even the neutral

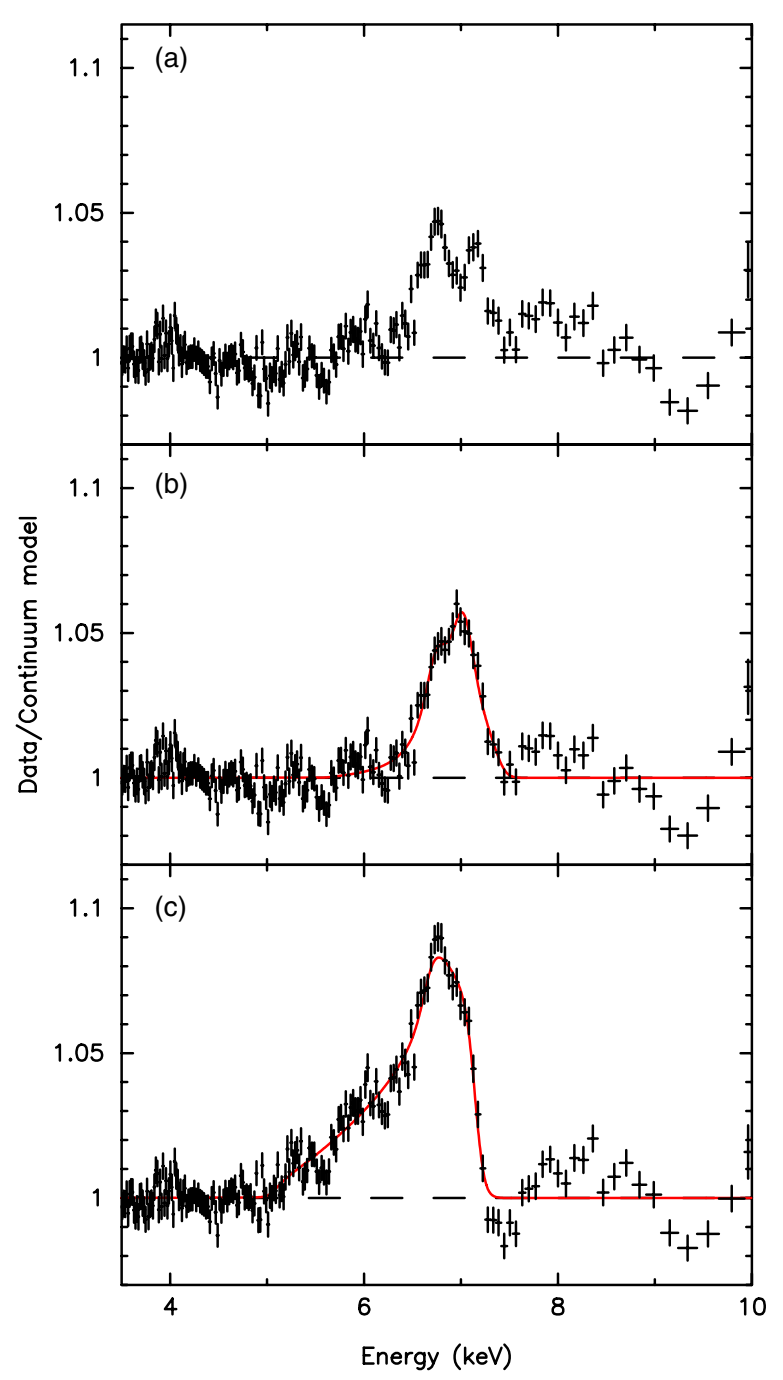

Figure 1. Ratio of data to continuum model for GX 340+0. Each panel shows the same spectrum, fit with a different model (see Table 2 for spectral fits). (a) The continuum is fit at $2.5-5 \mathrm{keV}$ and $8-10 \mathrm{keV}$, ignoring the $\mathrm{Fe} \mathrm{K}$ region, with an absorbed blackbody + disk blackbody model. A clear double-peaked structure is apparent. (b) An absorption line at $6.97 \mathrm{keV}$ is added to the model along with a relativistic Fe $\mathrm{K}$ emission line. The ratio shown is that of the data to the continuum + absorption line model, thus, showing the emission line profile only. The red, solid line shows the emission line model. (c) Here we follow D'Aì et al. (2009), allowing the ISM Fe abundance to vary, changing the depth of the Fe K edge from ISM absorption. The emission line model (red, solid line) is shown.

Fe K edge at $\sim 7 \mathrm{keV}$ is significant. Unfortunately, a large neutral $\mathrm{Fe} \mathrm{K}$ edge can be a big problem when fitting the Fe $\mathrm{K}$ emission line. If there is any small change in ISM Fe abundance toward the source, it will change the depth of the edge, and consequently the appearance of the emission line. Additionally, the edge is likely to be more complicated than the simple step-like function used in absorption models. Detailed structure has been observed for $\mathrm{Fe} \mathrm{L}$, and several $\mathrm{Ne}$ and $\mathrm{O}$ edges with sensitive grating spectra (e.g., Juett et al. 2004, 2006). Thus, with a large $N_{\mathrm{H}}$, structure in the edge might affect results.

When fitting these data, D'Aì et al. (2009) chose to allow the ISM Fe abundance to vary, effectively changing the size of the $\mathrm{Fe} \mathrm{K}$ edge. This produces a good fit, and the resulting $\mathrm{Fe} \mathrm{K}$ emission line profile looks good. We, similarly, achieve good fits by varying the Fe abundance. Nevertheless, there is not a unique model that fits the data well (see the line profiles in Figure 1). 
Table 2

Spectral Fitting Parameters for GX 340+0

\begin{tabular}{lcc}
\hline \hline \multicolumn{1}{c}{ Parameter } & Variable Fe Abundance & Fe XXVI Absorption Line \\
\hline$N_{H}\left(10^{22} \mathrm{~cm}^{-2}\right)$ & $10.9 \pm 0.1$ & $10.0 \pm 0.1$ \\
Fe abundance (rel. to solar) & $0.25_{-0.07}^{+0.03}$ & 1.0 (fixed) \\
Disk $k T(\mathrm{keV})$ & $1.64 \pm 0.04$ & $1.21 \pm 0.02$ \\
Disk normalization & $127 \pm 7$ & $325 \pm 9$ \\
Blackbody $k T(\mathrm{keV})$ & $2.44 \pm 0.09$ & $1.93 \pm 0.01$ \\
Bbody normalization $\left(10^{-2}\right)$ & $9.2 \pm 0.5$ & $15.2 \pm 0.1$ \\
Gaussian line energy $(\mathrm{keV})$ & $\ldots$ & 6.97 (frozen) \\
Gaussian $\sigma(\mathrm{keV})$ & $\ldots$ & 0.05 (frozen) \\
Gaussian normalization & $\ldots$ & $-1.1 \pm 0.1) \times 10^{-3}$ \\
Diskline line energy $(\mathrm{keV})$ & $6.97-0.01$ & $6.89 \pm 0.01$ \\
Emissivity, $q$ & $3.7 \pm 0.1$ & $1.85 \pm 0.14$ \\
$R_{\text {in }}\left(G M / c^{2}\right)$ & $9.3 \pm 0.2$ & $22 \pm 4$ \\
$i\left(^{\circ}\right)$ & $22 \pm 1$ & $37 \pm 4$ \\
Diskline normalization $\left(10^{-3}\right)$ & $13.1 \pm 0.2$ & $5.5 \pm 0.2$ \\
EW $(\mathrm{eV})$ & 80 & 38 \\
$\chi^{2} /$ dof & $1827 / 1548$ & $1925 / 1548$ \\
\hline
\end{tabular}

Notes. The outer disk radius was fixed at $1000 \mathrm{GM} / \mathrm{c}^{2}$ in both cases. Normalization of the disk blackbody is in $R_{\mathrm{inner}}^{2} \cos i /(D 10)^{2}$ where $R_{\text {inner }}$ is the inner disk radius in $\mathrm{km}, D 10$ is the distance to the source in units of $10 \mathrm{kpc}$, and $i$ is the disk inclination. Normalization of the blackbody component is $L 39 /(D 10)^{2}$, where $L 39$ is the luminosity in units of $10^{39} \mathrm{erg} \mathrm{s}^{-1}$. The normalization of both the Gaussian and the diskline is the line flux in photons $\mathrm{cm}^{-2} \mathrm{~s}^{-1}$.

Figure 1(a) shows the resulting line profile when the continuum is fit from 2.5 to $5 \mathrm{keV}$ and 8 to $10 \mathrm{keV}$, ignoring the $\mathrm{Fe} \mathrm{K}$ region (and assuming standard abundances). Evidently, the resulting residuals do not look like a typical $\mathrm{Fe} \mathrm{K}$ emission line profile, with a clear dip at around $7 \mathrm{keV}$. As mentioned above, D'Aì et al. (2009) successfully correct for this by assuming that the ISM Fe abundance toward this source is lower (see Figure 1(c)). With a lower $\mathrm{Fe}$ abundance, the absorption edge is weaker; thus the line profile no longer shows a dip.

Here, we alternatively suggest that this can also be interpreted as an Fe XXVI absorption line at $6.97 \mathrm{keV}$ superposed on a broad $\mathrm{Fe} \mathrm{K}$ emission line (see Figure 1(b) and Table 2). There are precedents for such a scenario. For instance, Boirin et al. (2005) find Fe XXV and Fe XXVI absorption lines superposed on a broad Fe $\mathrm{K}$ emission line in the dipping LMXB 4U 1323-62, and recently similar absorption lines superposed on a broad Fe K emission line were observed in the black hole binary GRO J1655-40 (Díaz Trigo et al. 2007).

As is apparent in Figure 1, the two models lead to differing emission-line profiles. While in the model with varying $\mathrm{Fe}$ abundance the line profile looks asymmetric, it is not as obviously asymmetric when including an absorption line. Fitting the emission line profiles with the diskline model we also achieve different measurements of $R_{\text {in }}: 9.3 \pm 0.2 G M / c^{2}$ (consistent with the analysis of D'Aì et al. 2009) compared to $22 \pm 4 G M / c^{2}$ for the alternative model with an absorption line included. Due to the ambiguous nature of the line profile in this source we do not consider this spectrum further here, though parameters from the above spectral fits can be found in Table 2.

$G X 349+2$. We fit the two Suzaku spectra separately, even though the observation times are close together, in order to investigate any variability. The Suzaku/PIN spectrum was fit from 12 to $25 \mathrm{keV}$. In the XMM-Newton spectrum, there are large residuals at $\sim 1 \mathrm{keV}$, which are well fit by a Gaussian emission line (Iaria et al. 2009).

Cyg $X$-2. XIS spectra fitted from 0.8 to $10 \mathrm{keV}$, ignoring 1.5-2.5 keV. The Suzaku/PIN spectrum was fit from 12 to $25 \mathrm{keV}$. We find large residuals at $\sim 1 \mathrm{keV}$ (in all XIS spectra), that are well fit by a single Gaussian emission line. This spectral feature has previously been observed in this source (e.g., Vrtilek et al. 1988; Piraino et al. 2002; Shaposhnikov et al. 2009), and may be a complex of blended emission lines (Vrtilek et al. 1988; Schulz et al. 2009).

SAX J1808.4-3658. We do not re-fit the Suzaku and XMMNewton spectra of this source here, and refer the reader to Cackett et al. (2009a) regarding specifics.

HETE J1900.1-2455. The Suzaku/PIN spectrum was fit at $12-30 \mathrm{keV}$. This spectrum is quite noisy, though an $\mathrm{Fe} \mathrm{K}$ emission line is apparent. A broad $(\sigma=0.8 \mathrm{keV})$ Gaussian fits the line well $\left(\chi^{2}=1148\right.$, dof $\left.=1228\right)$, and a relativistic disk line is not statistically required. We do, however, fit the diskline to allow comparison with other data, and it gives a similarly good fit $\left(\chi^{2}=1146\right.$, dof $\left.=1226\right)$. Also note that the continuum model for this source only requires a power-law component and no disk blackbody or blackbody is required.

\subsubsection{Investigating the Effects of Pile-up}

Of all the sources observed, Cyg X-2 had the highest count rate observed by $S u z a k u$, leading to severe pile-up. The continuum shape changes due to pile-up, becoming artificially harder (e.g., Davis 2001). In order to investigate the robustness of the $\mathrm{Fe} \mathrm{K}$ line profile in Cyg X-2, we extracted spectra from multiple different regions. In all cases, we used a box of $250 \times 400$ pixels, but had a circular exclusion region of varying radius centered on the source. The radius of this circle ranged from 0 pixels to 90 pixels. We fitted the spectra from 0.8 to $10 \mathrm{keV}$, ignoring the $1.5-2.5 \mathrm{keV}$ energy range, as usual. The spectra were allowed to have completely different continuum models, fit using an absorbed disk blackbody, plus a single temperature blackbody. Additionally, we had to include a narrow Gaussian at $1 \mathrm{keV}$. Here, we do not fit the PIN data; thus no power-law component is required. We do not fit the PIN data as pile-up significantly affects the shape of the XIS spectrum; hence the broadband spectrum will not fit well unless the XIS spectra are corrected for pile-up.

We found that even though each spectrum had different continuum shapes (due to pile-up effects), the Fe $\mathrm{K}$ line profile 

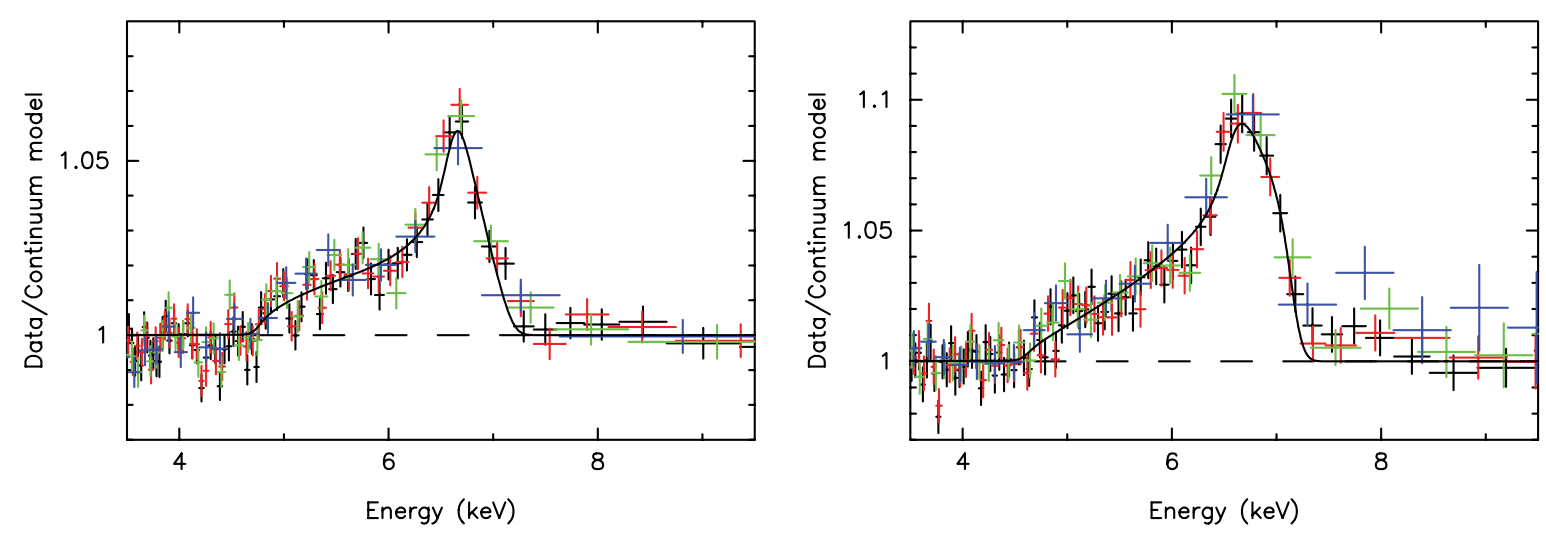

Figure 2. Left: Fe line profile for Cyg X-2, excluding a central circular region of varying radius. Black: exclusion circle radius $=0$ pixels, red: radius $=30$ pixels, green: radius $=60$ pixels, blue: radius $=90$ pixels. The Fe K line profile remains consistent regardless of the extraction region used. Right: Fe line profile for Ser X-1 from the Suzaku observation. Only data from XIS 3 are shown. Colors denote the same size extraction regions as for Cyg X-2.

Table 3

Investigating Pile-up in Cyg X-2: Spectral Parameters for Different Extraction Regions

\begin{tabular}{|c|c|c|c|c|}
\hline \multirow[t]{2}{*}{ Parameter } & \multicolumn{4}{|c|}{ Excluded Circle Radius } \\
\hline & 0 pixels & 30 pixels & 60 pixels & 90 pixels \\
\hline$N_{H}\left(10^{22} \mathrm{~cm}^{-2}\right)$ & $0.147 \pm 0.003$ & $0.136 \pm 0.004$ & $0.128 \pm 0.004$ & $0.130 \pm 0.005$ \\
\hline Disk $k T(\mathrm{keV})$ & $1.595 \pm 0.006$ & $1.555 \pm 0.002$ & $1.52 \pm 0.02$ & $1.45 \pm 0.04$ \\
\hline Disk normalization & $124 \pm 2$ & $151 \pm 1$ & $165 \pm 3$ & $190 \pm 20$ \\
\hline Blackbody $k T(\mathrm{keV})$ & $9 \pm 1$ & $4.1 \pm 0.1$ & $2.9 \pm 0.2$ & $2.2 \pm 0.2$ \\
\hline Bbody normalization $\left(10^{-2}\right)$ & $41 \pm 2$ & $7.0 \pm 0.1$ & $4.2 \pm 0.1$ & $4.4 \pm 0.6$ \\
\hline Line Energy (keV) & $6.97-0.02$ & $6.97-0.02$ & $6.97-0.02$ & $6.97-0.02$ \\
\hline Emissivity, $q$ & $5.8 \pm 0.6$ & $5.7 \pm 0.5$ & $6.3 \pm 0.8$ & $6.5_{-1.5}^{+1.0}$ \\
\hline$R_{\text {in }}\left(G M / c^{2}\right)$ & $8.0 \pm 0.2$ & $7.8 \pm 0.2$ & $7.9 \pm 0.3$ & $8.0 \pm 0.5$ \\
\hline$i\left(^{\circ}\right)$ & $24.0 \pm 0.5$ & $24.3 \pm 0.6$ & $23.9 \pm 0.6$ & $23 \pm 1$ \\
\hline Line normalization $\left(10^{-3}\right)$ & $7.2 \pm 0.4$ & $8.4 \pm 0.4$ & $8.7 \pm 0.6$ & $8.4 \pm 1.0$ \\
\hline $\mathrm{EW}(\mathrm{eV})$ & 69 & 76 & 79 & 74 \\
\hline
\end{tabular}

Notes. Spectral parameters from fitting the XIS 3 data. While the continuum is variable due to pile-up effects, the Fe $\mathrm{K}$ line parameters are remain consistent. The normalization of the disk blackbody is in $R_{\mathrm{inner}}^{2} \cos i /(D 10)^{2}$ where $R_{\text {inner }}$ is the inner disk radius in $\mathrm{km}, D 10$ is the distance to the source in units of 10 $\mathrm{kpc}$, and $i$ is the disk inclination. The normalization of the blackbody component is $L 39 /(D 10)^{2}$, where $L 39$ is the luminosity in units of $10^{39} \mathrm{erg} \mathrm{s}^{-1}$. The normalization of the diskline is the line flux in photons $\mathrm{cm}^{-2} \mathrm{~s}^{-1}$.

was remarkably robust. This is clearly demonstrated in Table 3 and Figure 2. In Table 3, we give the continuum and Fe K line parameters from fitting the spectra with different size exclusion regions. Figure 2 shows the resulting line profiles for each spectrum. The Fe K line is both quantitatively and qualitatively consistent, regardless of the pile-up correction. Thus, while with pile-up the continuum may vary significantly from the "true" (not piled-up) continuum shape (in the most extreme cases it is obvious that the continuum model parameters are not realistic), it appears that the $\mathrm{Fe} \mathrm{K}$ line shape can be robustly recovered as long as the continuum can be fit well. To further demonstrate this, we also show the Fe K profile from the Suzaku observation of Ser X-1 (Figure 2). We again see that the line profile remains consistent as the radius of the circular exclusion region is varied from 0 pixels to 90 pixels.

\subsubsection{Results from Phenomenological Fitting}

The spectral parameters from this phenomenological modeling are given in Tables 4 and 5 (the tables have been split into continuum and line parameters). Table 6 gives the $0.5-25 \mathrm{keV}$ unabsorbed fluxes and luminosities. A summary of all the Fe K lines observed in neutron star LMXBs can be seen in Figure 3.

The distribution of measured inner disk radii is shown in Figure 4 . We only use one measurement per source, choosing the one with the smallest fractional uncertainty. The vast majority of the measured inner disk radii fall into a relatively narrow range of 6-15 $G M / c^{2}$. Only two observations (of Ser X-1) fall outside this range, and even then the inner disk radii from those observations are only a little more than $1 \sigma$ away. Note that there are a number of fits in which $R_{\text {in }}$ pegs at the lower limit of the model (6 GM/ $\mathrm{c}^{2}$, the innermost stable circular orbit (ISCO) in the Schwarzschild metric). This corresponds to about $12 \mathrm{~km}$, assuming a $1.4 M_{\odot}$ neutron star. Given that this lower limit is very similar to predicted neutron star radii, smaller inner disk radii are not expected. The lines in the observations with $R_{\text {in }}=$ $6 \mathrm{GM} / \mathrm{c}^{2}$ are fit well by the model, and do not show large residuals in the red wing of the line (which would be expected if $R_{\text {in }}$ were smaller than measured).

One possibility for the large number of sources that reach the lower limit of the model is that there is some contribution to the broadening of the line from Compton scattering. Reis et al. (2009b) found that for 4U 1705-44 the measured inner disk radius increased when such effects were taken into account using reflection modeling.

We note that the differences in equivalent width (EW) between our original Suzaku analysis of Ser X-1, GX 349+2, and $4 \mathrm{U} 1820-30$ and this analysis can be attributed to updated responses. During the original analysis the standard response 
Table 4

Phenomenological Model Parameters: Continuum

\begin{tabular}{|c|c|c|c|c|c|c|c|c|c|c|c|c|}
\hline \multirow[t]{2}{*}{ Source } & \multirow[t]{2}{*}{ Mission } & \multirow[t]{2}{*}{ Obs. ID } & \multirow{2}{*}{$\begin{array}{c}N_{H} \\
10^{22}\left(\mathrm{~cm}^{-2}\right)\end{array}$} & \multicolumn{2}{|c|}{ Disk Blackbody } & \multicolumn{2}{|c|}{ Blackbody } & \multicolumn{2}{|c|}{ Power-law } & \multicolumn{3}{|c|}{ Gaussian } \\
\hline & & & & $k T_{\text {in }}(\mathrm{keV})$ & Norm. & $k T(\mathrm{keV})$ & Norm. $\left(10^{-2}\right)$ & $\Gamma$ & Norm. & Line Energy $(\mathrm{keV})$ & $\sigma(\mathrm{keV})$ & Norm. $\left(10^{-2}\right)$ \\
\hline \multirow[t]{4}{*}{ Serpens X-1 } & Suzaku & 401048010 & $0.67 \pm 0.04$ & $1.26 \pm 0.01$ & $97 \pm 5$ & $2.27 \pm 0.02$ & $5.23 \pm 0.06$ & $3.4 \pm 0.3$ & $0.9 \pm 0.2$ & & & \\
\hline & XMM-Newton & 0084020401 & $0.35 \pm 0.01$ & $0.95 \pm 0.01$ & $205 \pm 1$ & $1.76 \pm 0.01$ & $4.46 \pm 0.01$ & $\ldots$ & $\ldots$ & $1.10 \pm 0.01$ & $0.11 \pm 0.01$ & $1.3 \pm 0.01$ \\
\hline & $X M M$-Newton & 0084020501 & $0.35 \pm 0.01$ & $0.99 \pm 0.01$ & $175 \pm 3$ & $1.81 \pm 0.01$ & $3.13 \pm 0.02$ & $\ldots$ & $\ldots$ & $1.10 \pm 0.01$ & $0.14 \pm 0.01$ & $1.4 \pm 0.02$ \\
\hline & $X M M$-Newton & 0084020601 & $0.35 \pm 0.01$ & $0.95 \pm 0.01$ & $203 \pm 2$ & $1.76 \pm 0.01$ & $3.80 \pm 0.01$ & $\ldots$ & $\ldots$ & $1.10 \pm 0.01$ & $0.13 \pm 0.01$ & $1.2 \pm 0.01$ \\
\hline \multirow[t]{3}{*}{$4 \mathrm{U} 1636-53$} & XMM-Newton & 0303250201 & $0.36 \pm 0.01$ & $0.21 \pm 0.01$ & $(1.0 \pm 0.2) \times 10^{4}$ & $1.97 \pm 0.03$ & $0.15 \pm 0.01$ & $1.9 \pm 0.1$ & $0.22 \pm 0.01$ & $\ldots$ & $\ldots$ & $\ldots$ \\
\hline & $X M M$-Newton & 0500350301 & $0.51 \pm 0.01$ & $0.89 \pm 0.01$ & $101 \pm 4$ & $2.00 \pm 0.01$ & $1.19 \pm 0.01$ & $3.8 \pm 0.1$ & $0.54 \pm 0.01$ & $\ldots$ & $\ldots$ & $\ldots$ \\
\hline & XMM-Newton & 0500350401 & $0.50 \pm 0.01$ & $0.96 \pm 0.01$ & $103 \pm 4$ & $2.03 \pm 0.01$ & $1.57 \pm 0.01$ & $3.8 \pm 0.1$ & $0.58 \pm 0.01$ & $\ldots$ & $\ldots$ & $\ldots$ \\
\hline \multirow[t]{4}{*}{$4 \mathrm{U} 1705-44$} & Suzaku & 401046010 & 2.0 & $0.09 \pm 0.01$ & $\left(2.3_{-16}^{+7.2}\right) \times 10^{7}$ & $1.05 \pm 0.05$ & $0.063 \pm 0.005$ & $1.65 \pm 0.01$ & $0.124 \pm 0.002$ & $\ldots$ & $\ldots$ & $\ldots$ \\
\hline & Suzaku & 401046020 & $1.95 \pm 0.05$ & $1.16 \pm 0.02$ & $56 \pm 2$ & $2.12 \pm 0.03$ & $2.40 \pm 0.02$ & $2.85 \pm 0.08$ & $0.64 \pm 0.06$ & $\ldots$ & $\ldots$ & $\ldots$ \\
\hline & Suzaku & 401046030 & $2.02 \pm 0.05$ & $0.82 \pm 0.02$ & $82 \pm 5$ & $1.95 \pm 0.03$ & $0.95 \pm 0.01$ & $3.00 \pm 0.06$ & $0.49 \pm 0.03$ & $\ldots$ & $\ldots$ & $\ldots$ \\
\hline & $X M M$-Newton & 0402300201 & $1.53 \pm 0.01$ & $1.44 \pm 0.03$ & $2.5 \pm 0.2$ & $3.2 \pm 0.3$ & $0.25 \pm 0.01$ & & & $\ldots$ & $\ldots$ & $\ldots$ \\
\hline $4 \mathrm{U} 1820-30$ & Suzaku & 401047010 & $0.21 \pm 0.01$ & $1.18 \pm 0.01$ & $103 \pm 5$ & $2.44 \pm 0.01$ & $7.7 \pm 0.1$ & $2.3 \pm 0.1$ & $0.37 \pm 0.03$ & $\ldots$ & $\ldots$ & $\ldots$ \\
\hline \multirow[t]{2}{*}{ GX 17+2 } & Suzaku & 402050010 & $2.2 \pm 0.1$ & $1.75 \pm 0.02$ & $86 \pm 5$ & $2.66 \pm 0.02$ & $6.1 \pm 0.2$ & $2.6 \pm 0.6$ & $0.4 \pm 0.4$ & $\ldots$ & $\ldots$ & $\ldots$ \\
\hline & Suzaku & 402050020 & $2.14 \pm 0.05$ & $1.74 \pm 0.02$ & $92 \pm 4$ & $2.55 \pm 0.02$ & $9.2 \pm 0.3$ & $2.4 \pm 0.3$ & $0.2 \pm 0.2$ & $\ldots$ & $\ldots$ & $\ldots$ \\
\hline \multirow[t]{3}{*}{ GX $349+2$} & Suzaku & 400003010 & $0.77 \pm 0.01$ & $1.53 \pm 0.02$ & $112 \pm 4$ & $2.24 \pm 0.02$ & $12.5 \pm 0.2$ & $1.3 \pm 0.7$ & $0.01_{-0.01}^{+0.05}$ & $\ldots$ & $\ldots$ & $\ldots$ \\
\hline & Suzaku & 400003020 & $0.85 \pm 0.02$ & $1.48 \pm 0.02$ & $105 \pm 4$ & $2.30 \pm 0.01$ & $9.6 \pm 0.1$ & $2.3 \pm 0.1$ & $0.31 \pm 0.01$ & $\ldots$ & $\ldots$ & $\ldots$ \\
\hline & $X M M$-Newton & 0506110101 & $0.67 \pm 0.01$ & $1.17 \pm 0.01$ & $258 \pm 6$ & $1.85 \pm 0.01$ & $16.7 \pm 0.3$ & & & $1.08 \pm 0.01$ & $0.09 \pm 0.01$ & $3.9 \pm 0.4$ \\
\hline Cyg X-2 & Suzaku & 403063010 & $0.20 \pm 0.02$ & $1.50 \pm 0.01$ & $163 \pm 4$ & $2.21 \pm 0.02$ & $3.6 \pm 0.1$ & $2.8 \pm 0.1$ & $60 \pm 20$ & $1.01 \pm 0.01$ & $0.11 \pm 0.01$ & $9.0 \pm 0.6$ \\
\hline \multirow[t]{2}{*}{ SAX J1808.4-3658 } & Suzaku & 903003010 & $0.046 \pm 0.04$ & $0.48 \pm 0.01$ & $5.9 \pm 0.3$ & $1.00 \pm 0.02$ & $0.127 \pm 0.001$ & $1.93 \pm 0.01$ & $0.20 \pm 0.01$ & $\ldots$ & $\ldots$ & $\ldots$ \\
\hline & $X M M$-Newton & 0560180601 & $0.23 \pm 0.01$ & $0.23 \pm 0.01$ & $20400_{-5000}^{+13200}$ & $0.40 \pm 0.01$ & $0.125 \pm 0.004$ & $2.08 \pm 0.01$ & $0.33 \pm 0.01$ & $\ldots$ & $\ldots$ & $\ldots$ \\
\hline HETE J1900.1-2455 & Suzaku & 402016010 & $0.032 \pm 0.04$ & $\ldots$ & $\ldots$ & $\ldots$ & $\ldots$ & $2.14 \pm 0.01$ & $0.063 \pm 0.001$ & $\ldots$ & $\ldots$ & $\ldots$ \\
\hline
\end{tabular}

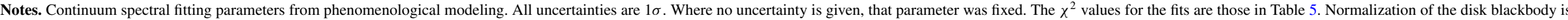

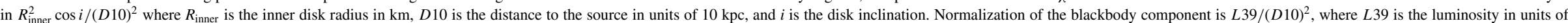
$10^{39} \mathrm{erg} \mathrm{s}^{-1}$. Normalization of the power-law component gives the photons $\mathrm{keV}^{-1} \mathrm{~cm}^{-2} \mathrm{~s}^{-1}$ at $1 \mathrm{keV}$. Finally, the normalization of the Gaussian is the total number of photons $\mathrm{cm}^{-2} \mathrm{~s}^{-1}$ in the line. 
Table 5

Phenomenological Model Parameters: Fe K Line

\begin{tabular}{|c|c|c|c|c|c|c|c|c|c|}
\hline \multirow[t]{2}{*}{ Source } & \multirow[t]{2}{*}{ Mission } & \multirow[t]{2}{*}{ Obs. ID } & \multicolumn{6}{|c|}{ Diskline } & \multirow[t]{2}{*}{$\chi^{2} /$ dof } \\
\hline & & & Line Energy (keV) & Emissivity Index & $R_{\text {in }}\left(G M / c^{2}\right)$ & Inclination $\left(^{\circ}\right)$ & Norm. $\left(10^{-3}\right)$ & EW $(\mathrm{eV})$ & \\
\hline \multirow[t]{4}{*}{ Serpens X-1 } & Suzaku & 401048010 & $6.97-0.02$ & $4.8 \pm 0.3$ & $8.0 \pm 0.3$ & $24 \pm 1$ & $5.6 \pm 0.4$ & 98 & $1442 / 1105$ \\
\hline & XMM-Newton & 0084020401 & $6.66 \pm 0.02$ & $1.8 \pm 0.2$ & $25 \pm 8$ & $32 \pm 3$ & $1.8 \pm 0.1$ & 43 & $2125 / 1831$ \\
\hline & XMM-Newton & 0084020501 & $6.97-0.03$ & $3.7 \pm 4$ & $14 \pm 1$ & $13 \pm 2$ & $1.6 \pm 0.1$ & 50 & $1961 / 1831$ \\
\hline & XMM-Newton & 0084020601 & $6.66 \pm 0.02$ & $2.0 \pm 0.2$ & $26 \pm 8$ & $27 \pm 2$ & $1.4 \pm 0.1$ & 38 & $2151 / 1831$ \\
\hline \multirow[t]{3}{*}{$4 \mathrm{U} 1636-53$} & XMM-Newton & 0303250201 & $6.4^{+0.02}$ & $2.7 \pm 0.1$ & $6.1^{+0.4}$ & $>80$ & $1.2 \pm 0.9$ & 184 & $1939 / 1832$ \\
\hline & XMM-Newton & 0500350301 & $6.4^{+0.04}$ & $2.8 \pm 0.1$ & $7.7 \pm 0.6$ & $>77$ & $1.2 \pm 0.1$ & 106 & $2112 / 1832$ \\
\hline & XMM-Newton & 0500350401 & $6.4^{+0.02}$ & $2.8 \pm 0.1$ & $6.0^{+0.2}$ & $>84$ & $2.5 \pm 0.1$ & 164 & $2406 / 1832$ \\
\hline \multirow[t]{4}{*}{$4 \mathrm{U} 1705-44$} & Suzaku & 401046010 & $6.62 \pm 0.03$ & 3.0 & 10.0 & 24 & $<0.44$ & $<76$ & $953 / 1122$ \\
\hline & Suzaku & 401046020 & $6.94 \pm 0.06$ & $4.8 \pm 0.6$ & $7.3 \pm 0.4$ & $24 \pm 4$ & $3.3 \pm 0.3$ & 120 & $1266 / 1120$ \\
\hline & Suzaku & 401046030 & $6.9 \pm 0.1$ & $3.7 \pm 0.3$ & $6^{+0.2}$ & $15 \pm 3$ & $1.3 \pm 0.1$ & 94 & $1152 / 1120$ \\
\hline & XMM-Newton & 0402300201 & $6.43 \pm 0.03$ & $2.1 \pm 0.1$ & $6^{+1}$ & $>74$ & $0.34 \pm 0.05$ & 151 & $1760 / 1752$ \\
\hline 4U 1820-30 & Suzaku & 401047010 & $6.97-0.03$ & $3.3 \pm 0.1$ & $6.0^{+0.1}$ & $<8$ & $2.6 \pm 0.3$ & 36 & $1269 / 1108$ \\
\hline \multirow[t]{2}{*}{ GX 17+2 } & Suzaku & 402050010 & $6.8 \pm 0.1$ & $2.6 \pm 0.2$ & $7 \pm 3$ & $15 \pm 15$ & $6.0 \pm 0.5$ & 46 & $636 / 606$ \\
\hline & Suzaku & 402050020 & $6.75 \pm 0.07$ & $4.6 \pm 0.7$ & $8.0 \pm 0.4$ & $27 \pm 2$ & $14 \pm 1$ & 81 & $628 / 606$ \\
\hline \multirow[t]{3}{*}{ GX $349+2$} & Suzaku & 400003010 & $6.97-0.01$ & $3.9 \pm 0.2$ & $7.5 \pm 0.4$ & $26 \pm 1$ & $16 \pm 1$ & 105 & $1268 / 1106$ \\
\hline & Suzaku & 400003020 & $6.64 \pm 0.03$ & $2.3 \pm 0.1$ & $10 \pm 2$ & $42 \pm 2$ & $9.2 \pm 0.6$ & 79 & $1262 / 1106$ \\
\hline & XMM-Newton & 0506110101 & $6.76 \pm 0.01$ & $1.9 \pm 0.1$ & $6.0^{+0.5}$ & $55 \pm 2$ & $12.1 \pm 0.3$ & 79 & $2531 / 1831$ \\
\hline Cyg X-2 & Suzaku & 403063010 & $6.97_{-0.02}$ & $6.5 \pm 1.3$ & $8.1 \pm 0.9$ & $25 \pm 3$ & $7.0 \pm 0.6$ & 65 & $1305 / 1129$ \\
\hline \multirow[t]{2}{*}{ SAX J1808.4-3658 } & Suzaku & 903003010 & $6.40^{+0.04}$ & $2.9 \pm 0.2$ & $12_{-1}^{+7}$ & $50_{-2}^{+7}$ & $0.70 \pm 0.06$ & 134 & $2987 / 2550$ \\
\hline & XMM-Newton & 0560180601 & $6.4^{+0.06}$ & $3.0 \pm 0.2$ & $13 \pm 4$ & $59 \pm 4$ & $0.68 \pm 0.04$ & 118 & $2124 / 1950$ \\
\hline HETE J1900.1-2455 & Suzaku & 402016010 & $6.97_{-0.2}$ & $5 \pm 1$ & $6^{+1}$ & $20 \pm 4$ & $0.16 \pm 0.02$ & 122 & $1146 / 1226$ \\
\hline
\end{tabular}

Notes. Fe K line parameters from phenomenological modeling. All uncertainties are $1 \sigma$. Where no uncertainty is given, that parameter was fixed. In the diskline model, the emissivity of the disk follows a power-law radial dependence, $R^{-q}$, where $q$ is the emissivity index we report here. The normalization of the diskline is the line flux in photons $\mathrm{cm}^{-2} \mathrm{~s}^{-1}$.

Table 6

Source Fluxes and Luminosities in the $0.5-25 \mathrm{keV}$ Range

\begin{tabular}{|c|c|c|c|c|c|c|}
\hline \multirow[t]{2}{*}{ Source } & \multirow[t]{2}{*}{ Mission } & \multirow[t]{2}{*}{ Obs. ID } & \multicolumn{2}{|c|}{ Phenomenological } & \multicolumn{2}{|c|}{ Reflection } \\
\hline & & & $\begin{array}{c}\text { Unabs. Flux } \\
\left(10^{-8} \mathrm{erg} \mathrm{s}^{-1} \mathrm{~cm}^{-2}\right)\end{array}$ & $\begin{array}{l}\text { Luminosity } \\
\left(10^{37} \mathrm{erg} \mathrm{s}^{-1}\right)\end{array}$ & $\begin{array}{c}\text { Unabs. Flux } \\
\left(10^{-8} \mathrm{erg} \mathrm{s}^{-1} \mathrm{~cm}^{-2}\right)\end{array}$ & $\begin{array}{c}\text { Luminosity } \\
\left(10^{37} \mathrm{erg} \mathrm{s}^{-1}\right)\end{array}$ \\
\hline \multirow[t]{4}{*}{ Serpens $X-1$} & Suzaku & 401048010 & $1.19 \pm 0.01$ & $10.0 \pm 5.0$ & $1.32 \pm 0.08$ & $11.1 \pm 5.6$ \\
\hline & XMM-Newton & 0084020401 & $0.70 \pm 0.01$ & $5.9 \pm 3.0$ & $0.71 \pm 0.01$ & $6.0 \pm 3.0$ \\
\hline & XMM-Newton & 0084020501 & $0.59 \pm 0.01$ & $5.0 \pm 2.5$ & $0.60 \pm 0.01$ & $5.1 \pm 2.5$ \\
\hline & XMM-Newton & 0084020601 & $0.65 \pm 0.01$ & $5.5 \pm 2.7$ & $0.66 \pm 0.01$ & $5.6 \pm 2.8$ \\
\hline \multirow[t]{3}{*}{$4 U$ 1636-53 } & XMM-Newton & 0303250201 & $0.17 \pm 0.01$ & $0.73 \pm 0.05$ & $0.18 \pm 0.02$ & $0.78 \pm 0.09$ \\
\hline & XMM-Newton & 0500350301 & $0.39 \pm 0.01$ & $1.7 \pm 0.1$ & $0.37 \pm 0.01$ & $1.6 \pm 0.1$ \\
\hline & XMM-Newton & 0500350401 & $0.48 \pm 0.01$ & $2.1 \pm 0.1$ & $0.46 \pm 0.03$ & $2.0 \pm 0.1$ \\
\hline \multirow[t]{4}{*}{$4 \mathrm{U} 1705-44$} & Suzaku & 401046010 & $0.17 \pm 0.01$ & $0.68 \pm 0.06$ & $0.57 \pm 0.05$ & $2.3 \pm 0.3$ \\
\hline & Suzaku & 401046020 & $0.61 \pm 0.01$ & $2.5 \pm 0.2$ & $0.49 \pm 0.01$ & $2.0 \pm 0.1$ \\
\hline & Suzaku & 401046030 & $0.30 \pm 0.01$ & $1.2 \pm 0.1$ & $0.31 \pm 0.01$ & $1.2 \pm 0.1$ \\
\hline & XMM-Newton & 0402300201 & $0.043 \pm 0.001$ & $0.17 \pm 0.01$ & $0.042 \pm 0.001$ & $0.17 \pm 0.01$ \\
\hline $4 \mathrm{U} 1820-30$ & Suzaku & 401047010 & $1.21 \pm 0.01$ & $8.4 \pm 0.9$ & $1.21 \pm 0.13$ & $8.4 \pm 1.3$ \\
\hline \multirow[t]{2}{*}{ GX $17+2$} & Suzaku & 402050010 & $2.37 \pm 0.01$ & $27.2 \pm 2.2$ & $2.27 \pm 0.01$ & $26.1 \pm 2.1$ \\
\hline & Suzaku & 402050020 & $2.60 \pm 0.01$ & $29.9 \pm 2.4$ & $2.62 \pm 0.01$ & $30.1 \pm 2.5$ \\
\hline \multirow[t]{3}{*}{ GX $349+2$} & Suzaku & 400003010 & $2.35 \pm 0.03$ & $7.0 \pm 3.5$ & $2.36 \pm 0.13$ & $7.1 \pm 3.6$ \\
\hline & Suzaku & 400003020 & $1.97 \pm 0.01$ & $5.9 \pm 2.9$ & $1.92 \pm 0.01$ & $5.7 \pm 2.9$ \\
\hline & XMM-Newton & 0506110101 & $2.38 \pm 0.01$ & $7.1 \pm 3.6$ & $2.40 \pm 0.09$ & $7.2 \pm 3.6$ \\
\hline Cyg X-2 & Suzaku & 403063010 & $2.22 \pm 0.01$ & $32.1 \pm 11.7$ & $2.30 \pm 0.01$ & $33.3 \pm 12.1$ \\
\hline \multirow[t]{2}{*}{ SAX J1808.4-3658 } & Suzaku & 903003010 & $0.20 \pm 0.01$ & $0.29 \pm 0.02$ & $0.27 \pm 0.02$ & $0.40 \pm 0.04$ \\
\hline & XMM-Newton & 0560180601 & $0.25 \pm 0.01$ & $0.37 \pm 0.03$ & $0.26 \pm 0.01$ & $0.38 \pm 0.03$ \\
\hline HETE J1900.1-2455 & Suzaku & 402016010 & $0.034 \pm 0.001$ & $0.053 \pm 0.015$ & $0.032 \pm 0.005$ & $0.050 \pm 0.016$ \\
\hline
\end{tabular}

Note. Distances used are given in Table 1.

matrices had to be used, which did not fully account for the window mode, and therefore gave incorrect fluxes.

\subsection{Reflection Models}

As noted earlier, the $\mathrm{Fe} \mathrm{K} \alpha$ emission line is only the most prominent feature expected from disk reflection where hard ionizing flux irradiates the accretion disk leading to line emission. In order to more self-consistently model the spectra, we fit reflection models which include line emission from important astrophysical metals (the strongest line being $\mathrm{Fe} \mathrm{K} \alpha$ ), as well as effects such as Compton broadening.

As is clear from the phenomenological modeling of the spectra (and as has been noted before, e.g., Cackett et al. 2008; Iaria et al. 2009), in the atoll and $Z$ sources, the blackbody component (potentially associated with the boundary 


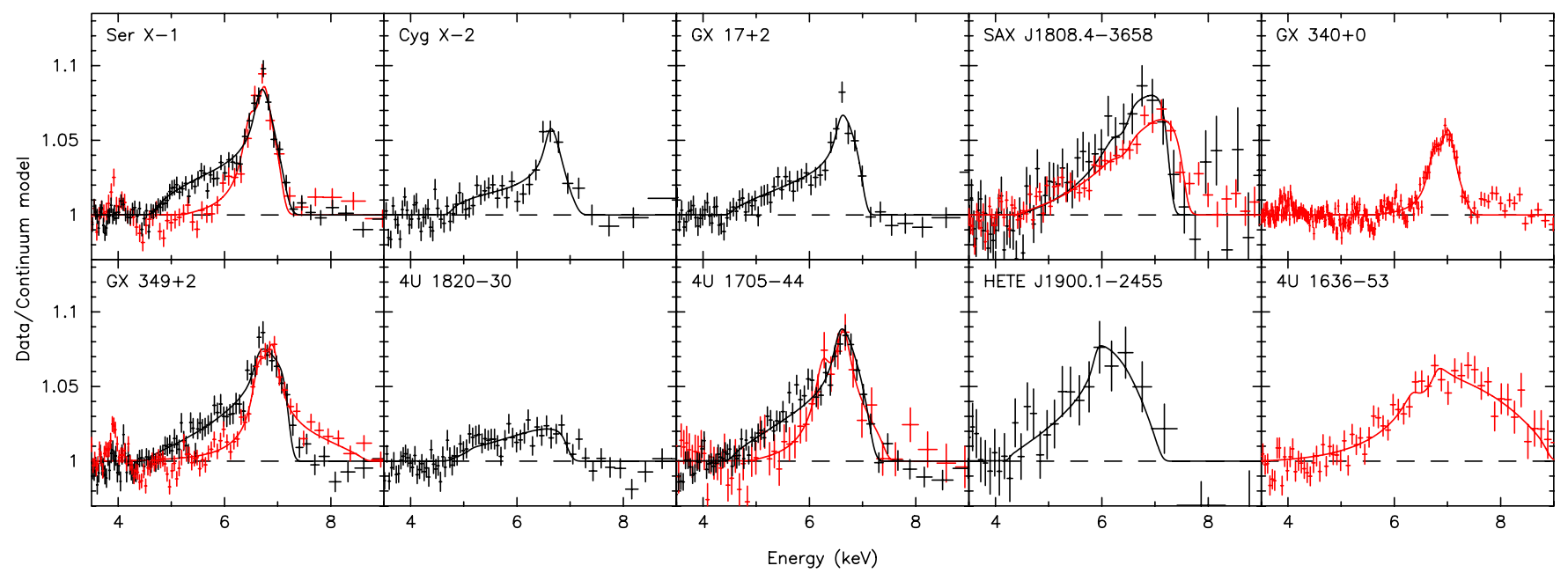

Figure 3. Summary of the Fe K emission lines in neutron star LMXBs. Plotted is the ratio of the data to the continuum model. The solid line shows the best-fitting diskline model. Data from Suzaku are shown in black (combined front-illuminated detectors), data from XMM-Newton is shown in red (PN camera).

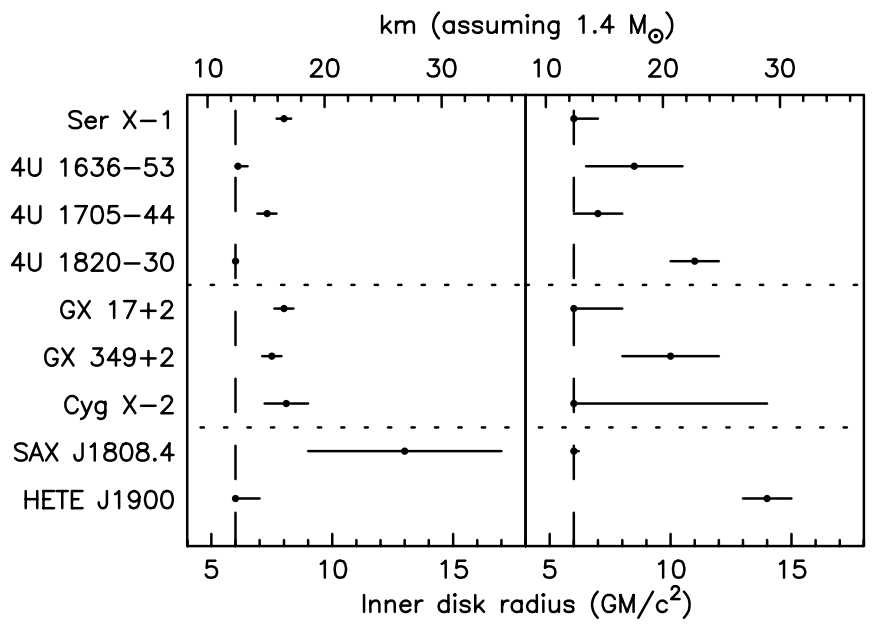

Figure 4. Measured inner disk radii $\left(G M / c^{2}\right)$ from phenomenological fits (left) and reflection fits (right). Where there are multiple observations, the one with the smallest fractional uncertainty was chosen. The upper $x$-axis shows the inner disk radius in $\mathrm{km}$ assuming a $1.4 M_{\odot}$ neutron star. The horizontal dotted lines separate the $\mathrm{Z}$ sources (top), atolls (middle) and accreting millisecond $\mathrm{X}$-ray pulsars (bottom). The vertical dashed line marks $6 G M / c^{2}$, the lower limit on the inner disk radius allowed by the model.

layer) is often dominating between 8 and $20 \mathrm{keV}$. Thus, it is the emission that provides the vast majority of ionizing flux that illuminates the accretion disk forming the $\mathrm{Fe} \mathrm{K}$ line (and reflection spectrum). In order to model the reflection component, one therefore needs to employ a reflection model where a blackbody component provides the illuminating flux (Ballantyne \& Strohmayer 2004; Ballantyne 2004). This differs from the standard reflection models used to model black hole sources (e.g., Ballantyne et al. 2001; Ross \& Fabian 2007), which assume illumination by a power-law spectrum. The resulting reflection spectra are quite different (see Figure 5 and Ballantyne 2004, for details), with the blackbody reflection model dropping off quickly above $10 \mathrm{keV}$ compared to the power-law reflection.

Here, we use models created to study the Fe $\mathrm{K}$ emission line observed in $4 \mathrm{U} 1820-30$ during a superburst, where a constant-density slab is illuminated by a blackbody ${ }^{9}$ (Ballantyne

9 The reflection models used here are to be made publicly available on the XSPEC Web site.

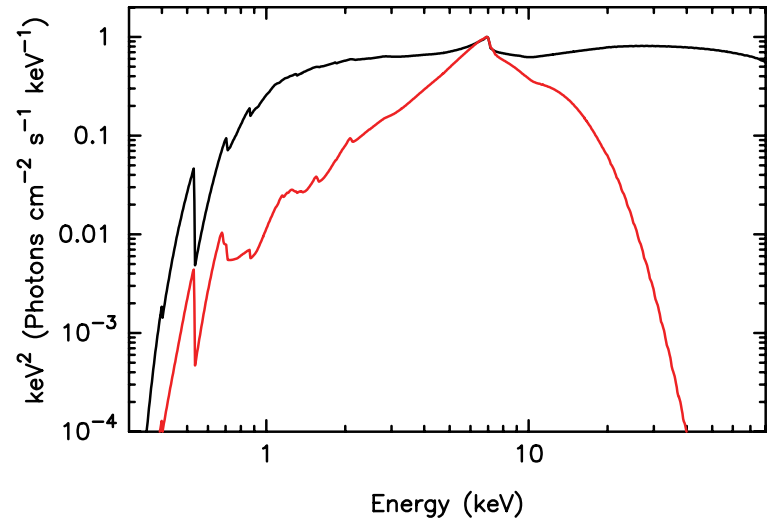

Figure 5. Blurred reflection models for illumination by a power law, spectral index $=2$ (black), and for illumination by a blackbody, $k T=2 \mathrm{keV}$ (red), both for an ionization parameter of 1000 (models used are from Ballantyne et al. 2001; Ballantyne 2004). The models are normalized so that they both peak at 1. The spectra are blurred using the rdblur model (a convolution with the diskline model), both assuming an emissivity index $q=3$, inner disk radius $=10 \mathrm{GM} / \mathrm{c}^{2}$, outer disk radius $=1000 \mathrm{GM} / \mathrm{c}^{2}$, and inclination $=30^{\circ}$. Both spectra are also absorbed assuming a quite typical value of $N_{\mathrm{H}}=5 \times 10^{21} \mathrm{~cm}^{-2}$.

\& Strohmayer 2004; Ballantyne 2004). We use the model assuming solar abundances for all sources except $4 \mathrm{U} 1820-30$, where we use the model for a $\mathrm{He}$ system (Ballantyne \& Strohmayer 2004). We note here that the density used in these models is $n_{\mathrm{H}}=10^{15} \mathrm{~cm}^{-3}$. This is significantly lower than the density expected at the inner disk in neutron star systems. Ballantyne (2004) tested increasing the density used in the model, and found that it did not significantly change the Fe K emission, though changes at lower energies $(<1 \mathrm{keV})$ were more important. While a higher density model would be more appropriate here, there is not yet one available for use.

We use a model where the irradiating blackbody component is included in the reflection model, in order to calculate the reflection fraction, $R$, which is given in Table 8 with respect to that for a point source above a slab. The overall model is given by a combination of the reflected spectrum and this blackbody. In the model, the ionization parameter is defined as

$$
\xi=\frac{4 \pi F_{\mathrm{x}}}{n_{\mathrm{H}}}
$$



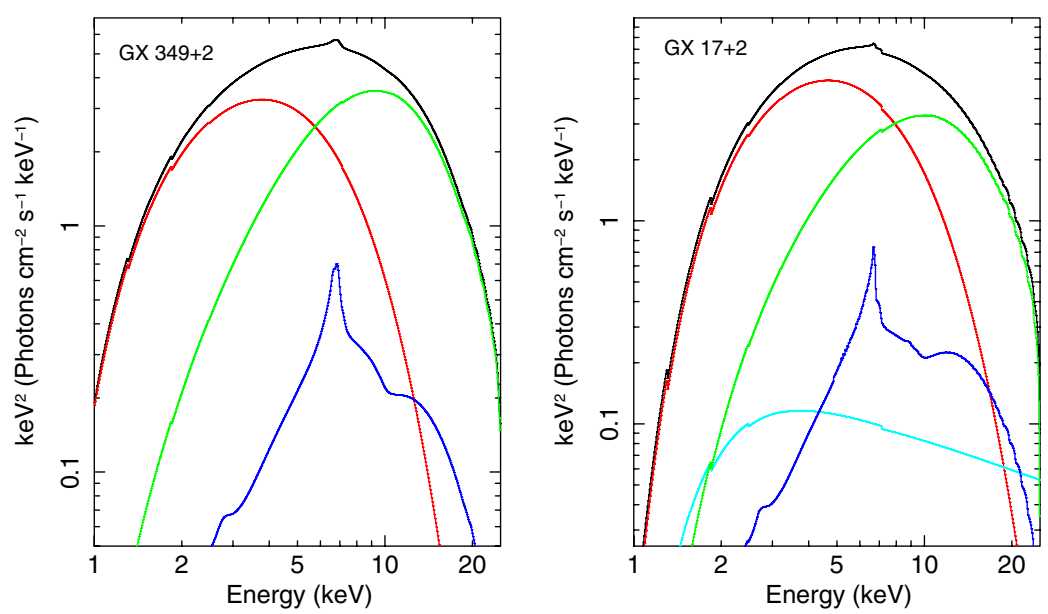

Figure 6. Reflection model fit to the second Suzaku observation of GX 349+2 (left) and the second Suzaku observation of GX 17+2 (right). The individual model components are shown as red, disk blackbody; green, blackbody; cyan, power law; blue, reflection component.

where $F_{\mathrm{x}}$ is the irradiating flux (erg $\mathrm{cm}^{-2} \mathrm{~s}^{-1}$ ) in the $0.001-100 \mathrm{keV}$ range and $n_{\mathrm{H}}$ is the hydrogen number density. The ionization parameter, $\xi$, therefore has units erg $\mathrm{cm} \mathrm{s}^{-1}$. The normalization of the reflection model is simply a scale factor that sets the overall strength of the combined blackbody and reflection spectrum, whereas the reflection fraction sets the relative strength of the two. The observed, unabsorbed flux (erg $\mathrm{cm}^{-2} \mathrm{~s}^{-1}$ ) of the blackbody plus reflection spectrum from the source in the $0.001-100 \mathrm{keV}$ range is therefore given by

$$
F_{\text {obs, unabs }}=\text { Norm } \times F_{\mathrm{x}}(1+R),
$$

where Norm is the normalization of the model. Rearranging gives

$$
\text { Norm }=\frac{4 \pi F_{\text {obs, unabs }}}{\xi n_{\mathrm{H}}(1+R)}
$$

and the density used in the models, $n_{\mathrm{H}}=10^{15} \mathrm{~cm}^{-3}$, is substituted.

To make these spectral fits more easily reproducible by other models we have calculated the equivalent blackbody normalization (using the definition of the XSPEC model bbody), from the best-fitting value of the reflection model normalization and $\xi$. The strength of the reflection component relative to this is given by the reflection fraction. So that the results are reproducible using the same model as used here, we also quote the normalization value directly from the model (as defined above).

The strength of the $\mathrm{Fe} \mathrm{K}$ line is dependent on both the ionization parameter and the temperature of the irradiating blackbody. As the blackbody temperature increases, the number of photons above the $\mathrm{Fe} \mathrm{K}$ edge increases, leading to a greater EW. The EW peaks both at low ionization parameters, $\log \xi=$ 1.0 , where $\mathrm{Fe} \mathrm{K} \alpha$ is at $6.4 \mathrm{keV}$, and then at $\log \xi \sim 2.7$ where the line is at $6.7 \mathrm{keV}$. At the peak of the higher ionization parameter, the $\mathrm{EW}$ is between $\sim 100$ and $300 \mathrm{eV}$ for a blackbody temperature in the range $1.5 \mathrm{keV}-3.0 \mathrm{keV}$. This is shown clearly in Figure 3 of Ballantyne (2004). These EWs are for a reflection fraction of 1. Lower reflection fractions lead to smaller EWs.

We relativistically blur this reflection model by convolving with the rdblur model in XSPEC. rdblur is simply a convolution with the diskline model, allowing the entire reflection component to be blurred by the relativistic effects present at the inner accretion disk. This blurred reflection component is then included in the model instead of the diskline model used in the phenomenological fits. This modeling acts to self-consistently model the entire reflection spectrum (multiple emission lines and continuum) rather than just treating the $\mathrm{Fe} \mathrm{K}$ line alone.

We are able to achieve good fits with this reflection modeling, generally finding similar inner disk radii as with phenomenological modeling (see spectral fit parameters in Tables 7 and 8). An example of our reflection fits is shown in Figure 6. It is important to note that because the blackbody component drops off over 8-20 keV, so too does the reflection component. The typical strong Compton hump seen in the black hole sources (both in active galactic nucleus and X-ray binaries) is therefore not apparent because of this.

The two sources where we do not fit this blackbody reflection model are the accreting millisecond pulsars (SAX J1808.4-3658 and HETE J1900.1-2455). Both sources show particularly hard spectra, where the high-energy continuum is dominated by a power law. We therefore fit these spectra with the constant density-ionized disk reflection model (Ballantyne et al. 2001), where a power law irradiates a slab. As with the blackbody reflection model, the model normalization is dependent on $\xi$, and is given by the same definition as above. We therefore have calculated the equivalent power-law normalization at $1 \mathrm{keV}$ so that these results are reproducible using other reflection models. Again, we also report the best-fitting normalization directly from the model so that these results can be easily reproduced using the same model. This model fits the data well in both cases. HETE J1900.1 - 2455 requires only the power-law reflection component, and no other continuum components are needed. SAX J1808.4-3658, on the other hand, requires both a disk blackbody and a blackbody in addition to the power-law reflection component.

All spectral parameters from this reflection fitting are given in Tables 7 and 8, and source fluxes and luminosities are given in Table 6. We also show the range of measured inner disk radii from the reflection component in Figure 4. We find that a large number of sources have their measured inner disk radii pegged at the model limit of $6 G M / c^{2}$, which we discuss in more detail later.

\section{SIMULTANEOUS TIMING OBSERVATIONS}

Fe $\mathrm{K}$ emission lines have been detected simultaneously with $\mathrm{kHz}$ QPOs previously (e.g., GX 17+2; Cackett et al. 2009b); however, there has not yet been the sensitivity to test whether the line and $\mathrm{kHz} \mathrm{QPO}$ originate from the same 
Table 7

Reflection Model Parameters: Continuum

\begin{tabular}{|c|c|c|c|c|c|c|c|c|c|c|c|c|}
\hline \multirow[t]{2}{*}{ Source } & \multirow[t]{2}{*}{ Mission } & \multirow[t]{2}{*}{ Obs. ID } & \multirow{2}{*}{$\begin{array}{c}N_{H} \\
\left(10^{22} \mathrm{~cm}^{-2}\right)\end{array}$} & \multicolumn{2}{|c|}{ Disk Blackbody } & \multicolumn{2}{|c|}{ Blackbody } & \multicolumn{2}{|c|}{ Power-law } & \multicolumn{3}{|c|}{ Gaussian } \\
\hline & & & & $k T_{\text {in }}(\mathrm{keV})$ & Norm. & $k T(\mathrm{keV})$ & Norm. $\left(10^{-2}\right)$ & $\Gamma$ & Norm. & Line Energy (keV) & $\sigma(\mathrm{keV})$ & Norm. $\left(10^{-2}\right)$ \\
\hline \multirow[t]{4}{*}{ Serpens X-1 } & Suzaku & 401048010 & $0.75 \pm 0.02$ & $1.22 \pm 0.01$ & $110 \pm 5$ & $2.29 \pm 0.01$ & $5.3 \pm 0.5$ & $3.80 \pm 0.11$ & $1.19 \pm 0.01$ & & $\ldots$ & \\
\hline & XMM-Newton & 0084020401 & $0.35 \pm 0.01$ & $0.97 \pm 0.01$ & $192 \pm 4$ & $1.79 \pm 0.01$ & $4.2 \pm 0.2$ & $\ldots$ & $\ldots$ & $1.09 \pm 0.01$ & $0.13 \pm 0.01$ & $1.4 \pm 0.1$ \\
\hline & XMM-Newton & 0084020501 & $0.35 \pm 0.01$ & $1.01 \pm 0.01$ & $166 \pm 6$ & $1.88 \pm 0.01$ & $2.95 \pm 0.07$ & $\ldots$ & $\ldots$ & $1.10 \pm 0.01$ & $0.17 \pm 0.01$ & $1.7 \pm 0.1$ \\
\hline & XMM-Newton & 0084020601 & $0.37 \pm 0.01$ & $0.99 \pm 0.01$ & $180 \pm 1$ & $1.82 \pm 0.01$ & $3.53 \pm 0.06$ & $\ldots$ & $\ldots$ & $0.95 \pm 0.01$ & $0.29 \pm 0.01$ & $4.6 \pm 0.1$ \\
\hline \multirow[t]{3}{*}{ 4U $1636-53$} & XMM-Newton & 0303250201 & $0.37 \pm 0.01$ & $0.21 \pm 0.01$ & $10500 \pm 2000$ & $1.92 \pm 0.02$ & $0.06 \pm 0.02$ & $1.94 \pm 0.06$ & $0.215 \pm 0.004$ & $\ldots$ & $\ldots$ & $\ldots$ \\
\hline & $X M M$-Newton & 0500350301 & $0.48 \pm 0.01$ & $0.93 \pm 0.01$ & $78 \pm 6$ & $2.13 \pm 0.04$ & $0.9 \pm 0.3$ & $3.5 \pm 0.1$ & $0.51 \pm 0.01$ & $\ldots$ & $\ldots$ & $\ldots$ \\
\hline & XMM-Newton & 0500350401 & $0.47 \pm 0.01$ & $1.00 \pm 0.01$ & $84 \pm 2$ & $2.10 \pm 0.01$ & $1.3 \pm 0.2$ & $3.5 \pm 0.1$ & $0.52 \pm 0.01$ & $\ldots$ & $\ldots$ & $\ldots$ \\
\hline \multirow[t]{4}{*}{ 4U 1705-44 } & Suzaku & 401046010 & 2.0 & $0.08 \pm 0.01$ & $\left(2.6_{-19}^{+3.4}\right) \times 10^{8}$ & $1.34 \pm 0.15$ & $0.016_{-0.006}^{+0.026}$ & $1.68 \pm 0.01$ & $0.13 \pm 0.03$ & $\ldots$ & $\ldots$ & $\ldots$ \\
\hline & Suzaku & 401046020 & $1.67 \pm 0.01$ & $1.04 \pm 0.01$ & $107 \pm 4$ & $2.42 \pm 0.02$ & $2.3 \pm 0.4$ & $\ldots$ & & $\ldots$ & $\ldots$ & $\ldots$ \\
\hline & Suzaku & 401046030 & $2.02 \pm 0.02$ & $0.81 \pm 0.01$ & $89 \pm 12$ & $2.12 \pm 0.01$ & $0.90 \pm 0.07$ & $3.02 \pm 0.04$ & $0.47 \pm 0.05$ & $\ldots$ & $\ldots$ & $\ldots$ \\
\hline & XMM-Newton & 0402300201 & $1.55 \pm 0.01$ & $1.42 \pm 0.01$ & $2.63 \pm 0.11$ & $3.15_{-0.05}$ & $0.22 \pm 0.06$ & $\ldots$ & 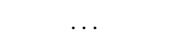 & $\ldots$ & $\ldots$ & $\ldots$ \\
\hline $4 \mathrm{U} 1820-30$ & Suzaku & 401047010 & $0.21 \pm 0.01$ & $1.14 \pm 0.01$ & $119 \pm 4$ & $2.50 \pm 0.04$ & $8.6 \pm 0.2$ & $2.38 \pm 0.05$ & $0.32 \pm 0.30$ & $\ldots$ & $\ldots$ & $\ldots$ \\
\hline \multirow[t]{2}{*}{ GX $17+2$} & Suzaku & 402050010 & $2.10 \pm 0.06$ & $1.69 \pm 0.02$ & $99 \pm 5$ & $2.65 \pm 0.02$ & $6.7 \pm 1.3$ & $1.8 \pm 0.5$ & $0.017_{-0.009}^{+0.211}$ & $\ldots$ & $\ldots$ & $\ldots$ \\
\hline & Suzaku & 402050020 & $2.15 \pm 0.05$ & $1.72 \pm 0.03$ & $95 \pm 4$ & $2.59 \pm 0.01$ & $9.4 \pm 1.3$ & $2.5 \pm 0.2$ & $0.27_{-0.15}^{+0.29}$ & $\ldots$ & $\ldots$ & $\ldots$ \\
\hline \multirow[t]{3}{*}{ GX $349+2$} & Suzaku & 400003010 & $0.77 \pm 0.01$ & $1.55 \pm 0.01$ & $109 \pm 4$ & $2.33 \pm 0.01$ & $11.5 \pm 2.1$ & $\ldots$ & $\ldots$ & $\ldots$ & $\ldots$ & $\ldots$ \\
\hline & Suzaku & 400003020 & $0.80 \pm 0.01$ & $1.47 \pm 0.01$ & $112 \pm 4$ & $2.35 \pm 0.01$ & $8.9 \pm 0.8$ & $\ldots$ & $\ldots$ & $\ldots$ & $\ldots$ & $\ldots$ \\
\hline & XMM-Newton & 0506110101 & $0.67 \pm 0.01$ & $1.24 \pm 0.01$ & $221 \pm 6$ & $1.89 \pm 0.01$ & $15.1 \pm 0.1$ & $\ldots$ & $\ldots$ & $1.07 \pm 0.01$ & $0.09 \pm 0.01$ & $3.4 \pm 0.1$ \\
\hline Cyg X-2 & Suzaku & 403063010 & $0.24 \pm 0.01$ & $1.50 \pm 0.01$ & $163 \pm 1$ & $2.23 \pm 0.01$ & $3.5 \pm 0.2$ & $2.93 \pm 0.04$ & $0.87 \pm 0.09$ & $1.00 \pm 0.01$ & $0.11 \pm 0.01$ & $10 \pm 1$ \\
\hline \multirow[t]{2}{*}{ SAX J1808.4-3658 } & Suzaku & 903003010 & $0.30 \pm 0.01$ & $0.41 \pm 0.01$ & $1370 \pm 120$ & $0.91 \pm 0.01$ & $0.05 \pm 0.01$ & $2.14 \pm 0.02$ & $0.27 \pm 0.01$ & $\ldots$ & $\ldots$ & $\ldots$ \\
\hline & $X M M$-Newton & 0560180601 & $0.27 \pm 0.02$ & $0.48 \pm 0.01$ & $232 \pm 21$ & $0.19 \pm 0.01$ & $0.6 \pm 0.1$ & $2.09 \pm 0.05$ & $0.31 \pm 0.01$ & $\ldots$ & $\ldots$ & $\ldots$ \\
\hline HETE J1900.1-2455 & Suzaku & 402016010 & $0.09 \pm 0.01$ & $\ldots$ & $\ldots$ & $\ldots$ & $\ldots$ & $2.18 \pm 0.01$ & $0.07 \pm 0.01$ & $\ldots$ & $\ldots$ & $\ldots$ \\
\hline
\end{tabular}

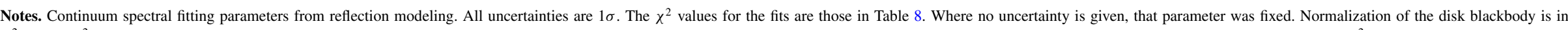

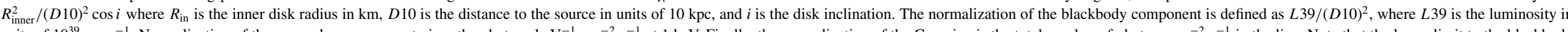

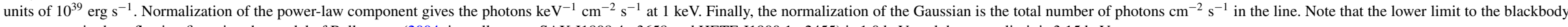
temperature in the reflection fits using the model of Ballantyne (2004; i.e., all except SAX J1808.4-3658 and HETE J1900.1-2455) is $1.0 \mathrm{keV}$, and the upper limit is $3.15 \mathrm{keV}$. 
Table 8

Reflection Model Parameters

\begin{tabular}{|c|c|c|c|c|c|c|c|c|c|}
\hline \multirow[t]{2}{*}{ Source } & \multirow[t]{2}{*}{ Mission } & \multirow[t]{2}{*}{ Obs. ID } & \multicolumn{6}{|c|}{ Blurred Reflection Parameters } & \multirow[t]{2}{*}{$\chi^{2} /$ dof } \\
\hline & & & Emissivity Index & $R_{\text {in }}\left(G M / c^{2}\right)$ & Inclination $\left(^{\circ}\right)$ & $\log \xi$ & Norm. $\left(10^{-26}\right)$ & Refl. Fraction & \\
\hline \multirow[t]{4}{*}{ Serpens X-1 } & Suzaku & 401048010 & $2.2 \pm 0.1$ & $6^{+1}$ & $16 \pm 1$ & $2.6 \pm 0.1$ & $13.6 \pm 1.3$ & $0.18 \pm 0.01$ & $1526 / 1105$ \\
\hline & XMM-Newton & 0084020401 & $>6$ & $107 \pm 11$ & $5 \pm 2$ & $2.8 \pm 0.1$ & $7.5 \pm 0.3$ & $0.10 \pm 0.01$ & $2213 / 1831$ \\
\hline & XMM-Newton & 0084020501 & $2.2 \pm 0.1$ & $15 \pm 2$ & $9 \pm 2$ & $2.6 \pm 0.1$ & $8.2 \pm 0.2$ & $0.16 \pm 0.01$ & $1982 / 1831$ \\
\hline & XMM-Newton & 0084020601 & $2.9 \pm 0.1$ & $44 \pm 2$ & $<3$ & $2.8 \pm 0.1$ & $6.4 \pm 0.1$ & $0.11 \pm 0.01$ & $2199 / 1831$ \\
\hline \multirow[t]{3}{*}{$4 U 1636-53$} & XMM-Newton & 0303250201 & $2.6 \pm 0.1$ & $8.5 \pm 2.0$ & $>68$ & $2.5 \pm 0.1$ & $0.20 \pm 0.05$ & $5.0_{-0.5}$ & $1881 / 1832$ \\
\hline & XMM-Newton & 0500350301 & $3.2 \pm 0.4$ & $13 \pm 4$ & $63_{-7}^{+16}$ & $3.2 \pm 0.1$ & $0.54 \pm 0.20$ & $0.52 \pm 0.08$ & $2080 / 1832$ \\
\hline & XMM-Newton & 0500350401 & $2.9 \pm 0.1$ & $13 \pm 2$ & $>75$ & $3.0 \pm 0.1$ & $1.3 \pm 0.2$ & $0.42 \pm 0.02$ & $2339 / 1832$ \\
\hline \multirow[t]{4}{*}{$4 \mathrm{U} 1705-44$} & Suzaku & 401046010 & 3.0 & 10.0 & 24 & $3.0 \pm 0.2$ & $0.017_{-0.007}^{+0.028}$ & $5.0_{-2.5}$ & $1042 / 1127$ \\
\hline & Suzaku & 401046020 & $6.0 \pm 0.8$ & $7 \pm 1$ & $32 \pm 1$ & $3.0 \pm 0.1$ & $3.0 \pm 0.5$ & $0.43 \pm 0.02$ & $1387 / 1124$ \\
\hline & Suzaku & 401046030 & $5.5 \pm 2.2$ & $11 \pm 6$ & $<4$ & $2.0 \pm 0.1$ & $8.7 \pm 0.7$ & $1.1 \pm 0.1$ & $1135 / 1122$ \\
\hline & XMM-Newton & 0402300201 & $>3.4$ & $34_{-12}^{+5}$ & $16 \pm 3$ & $3.0 \pm 0.1$ & $0.26 \pm 0.07$ & $0.21 \pm 0.02$ & $1766 / 1752$ \\
\hline $4 U$ 1820-30 & Suzaku & 401047010 & $>6$ & $11 \pm 1$ & $27 \pm 2$ & $2.6 \pm 0.1$ & $28.1 \pm 0.5$ & $0.11 \pm 0.02$ & $1336 / 1110$ \\
\hline \multirow[t]{2}{*}{$\mathrm{GX} 17+2$} & Suzaku & 402050010 & $2.2 \pm 0.1$ & $6^{+2}$ & $<11$ & $2.7 \pm 0.1$ & $15.5 \pm 2.9$ & $0.20 \pm 0.03$ & $647 / 606$ \\
\hline & Suzaku & 402050020 & $2.3 \pm 0.3$ & $6^{+2}$ & $<6$ & $2.7 \pm 0.1$ & $20.0 \pm 2.7$ & $0.21 \pm 0.02$ & $684 / 606$ \\
\hline \multirow[t]{3}{*}{ GX $349+2$} & Suzaku & 400003010 & $2.4 \pm 0.1$ & $10 \pm 2$ & $28 \pm 1$ & $2.9 \pm 0.1$ & $15.0 \pm 2.7$ & $0.24 \pm 0.01$ & $1341 / 1109$ \\
\hline & Suzaku & 400003020 & $>1.8$ & $386 \pm 97$ & $18 \pm 2$ & $3.1 \pm 0.1$ & $7.7 \pm 0.7$ & $0.22 \pm 0.01$ & $1276 / 1109$ \\
\hline & XMM-Newton & 0506110101 & $0.3 \pm 0.4$ & $6^{+54}$ & $48 \pm 2$ & $2.9 \pm 0.1$ & $22.3 \pm 0.1$ & $0.14 \pm 0.01$ & $2500 / 1831$ \\
\hline Cyg X-2 & Suzaku & 403063010 & $2.0 \pm 0.1$ & $6^{+8}$ & $<9$ & $2.6 \pm 0.1$ & $9.5 \pm 0.4$ & $0.31 \pm 0.03$ & $1379 / 1135$ \\
\hline \multirow[t]{2}{*}{ SAX J1808.4-3658 } & Suzaku & 903003010 & $>5.2$ & $14 \pm 2$ & $67-4$ & $2.7 \pm 0.1$ & $10.2 \pm 0.5$ & $0.86 \pm 0.08$ & $581 / 620$ \\
\hline & XMM-Newton & 0560180601 & $2.2 \pm 0.1$ & $6.0^{+0.2}$ & $67_{-1}$ & $2.8 \pm 0.1$ & $8.6 \pm 0.2$ & $0.34 \pm 0.03$ & $2120 / 1952$ \\
\hline HETE J1900.1-2455 & Suzaku & 402016010 & $5.2 \pm 0.8$ & $14 \pm 1$ & $<4$ & $2.8 \pm 0.1$ & $2.1 \pm 0.3$ & $0.31 \pm 0.03$ & $1101 / 1227$ \\
\hline
\end{tabular}

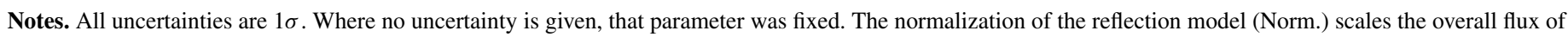
this component. See Section 4.2 for further discussion.

region. While simultaneity is clearly important due to known changes in $\mathrm{kHz}$ QPO frequency with source state, comparisons of non-simultaneous measurements of $R_{\text {in }}$ from Fe $\mathrm{K}$ lines and $\mathrm{kHz}$ QPOs are promising (Cackett et al. 2008). Simultaneous observations were performed by the RXTE Proportional Counter Array for both the Suzaku pointings of GX 17+2 and the second Cyg X-2 observations. Both sources are known kHz QPO sources. Those QPOs were previously reported by Wijnands et al. (1997) and Homan et al. (2002) for GX 17+2, and by Wijnands et al. (1998) for Cyg X-2.

We have analyzed the PCA observations, considering segments of continuous observation (Obs. IDs): two and five Obs. IDs worth of data were recorded for GX 17+2 and Cyg X-2, respectively. For each Obs. ID, we have computed an average power density spectrum (PDS) with a $1 \mathrm{~Hz}$ resolution, using events recorded between 2 and $40 \mathrm{keV}$. The PDSs are normalized according to Leahy et al. (1983), so that in the absence of significant dead time (as is the case here for both sources), the Poisson noise level is flat and constant with a value close to 2. The PDS so computed was then blindly searched for excess power between $300 \mathrm{~Hz}$ and $1400 \mathrm{~Hz}$ using a scanning technique, as presented in Boirin et al. (2000). We failed to detect any $\mathrm{kHz}$ QPOs in any of the observations, for both sources. The analysis was also performed considering photons of energy between 5 and $40 \mathrm{keV}$, but no $\mathrm{kHz}$ QPOs were detected. Finally, we have also produced an averaged PDS containing all observations. No QPOs were detected either.

We have derived upper limits for QPOs of various widths, as listed in Table 9 for each pointed observation. Using data from combined observations, the upper limits are $15.5 \%$ and $17.1 \%$ for GX $17+2$, and $4.6 \%$ and $5.1 \%$ for Cyg X-2 for signal of 100 and $150 \mathrm{~Hz}$ widths, respectively. These upper limits are not constraining for GX 17+2, whose lower and upper $\mathrm{kHz}$ QPOs have maximum rms amplitudes of $\sim 4 \%$ and $\sim 6 \%$, respectively (Wijnands et al. 1997; Homan et al. 2002). This is explained by the combination of a very low source count rate and a reduced number of operating PCA units (2 or 3). For Cyg X-2, which displays larger source count rates, the rms upper limits are significantly lower, but unfortunately still above the maximum rms amplitude of its $\mathrm{kHz}$ QPO reported so far (Wijnands et al. 1998).

Nevertheless for Cyg X-2, inspecting lower frequencies, features around 5 and $50 \mathrm{~Hz}$ are visible in the Obs. ID averaged PDS. These are likely normal branch and horizontal branch QPOs (HBOs), previously known from the source. It has been shown in sources such as Cyg X-2 (the prototype being GX 17+2, Wijnands et al. 1997; Homan et al. 2002) that the upper $\mathrm{kHz}$ QPO frequency correlates with the HBO frequency up to a certain frequency where the upper QPO frequency keeps increasing while the $\mathrm{HBO}$ one saturates (see, for instance, Figure 5 in Psaltis et al. 1999). In Cyg X-2, the HBO frequency has been shown to increase from about $35 \mathrm{~Hz}$ up to a saturating frequency of $55 \mathrm{~Hz}$. At the same time, the upper QPO frequency varied from $\sim 730 \mathrm{~Hz}$ to $\sim 1000 \mathrm{~Hz}$, while the corresponding $\mathrm{HBO}$ frequency ranged from 35 to $55 \mathrm{~Hz}$ (Wijnands et al. 1998). According to Psaltis et al. (1999, their Figure 8), before the saturation, a power-law relationship exists between the HBO and upper QPO frequencies, in the form of $v_{\mathrm{HBO}}=13.2 \mathrm{a}_{1}\left(v_{\text {upper }} / 1000\right)^{\mathrm{b}_{1}}$ $\mathrm{Hz}$, with $a_{1} \sim 4.3$ and $b_{1} \sim 1.4$ in the case of Cyg X-2. The $\mathrm{HBO}$ QPO significance is $\sim 5 \sigma$ significance (single trial). Fitting the 1-2048 Hz PDS with two Lorentzians and a constant to account for the Poisson noise level, we have estimated the HBO frequency to be $49.5_{-2.4}^{+2.5} \mathrm{~Hz}$, the QPO width as $7.0_{-4.1}^{+4.3} \mathrm{~Hz}$ for an rms amplitude of about $3.5_{-1.5}^{+0.7} \%$. The latter two parameters are fully consistent with previous detections (Wijnands et al. 1998). Despite its low significance, it is therefore tempting to use the $\sim 50 \mathrm{~Hz} \mathrm{HBO}$ reported here (which is below the saturating frequency) to estimate with the above formula, the frequency of the upper QPO, that we failed to detect. This yields a frequency of about $910 \mathrm{~Hz}$. 
Table 9

RXTE Observations of GX 17+2, Cyg X-2, and HETE J1900.1-2455

\begin{tabular}{|c|c|c|c|c|c|c|c|c|c|c|}
\hline Name & Obs. ID & Date & Time & $T_{\mathrm{obs}}$ & Rate & Bkg. & $N_{\mathrm{PCU}}$ & $\mathrm{rms}_{50}(\%)$ & $\mathrm{rms}_{100}(\%)$ & $\operatorname{rms}_{150}(\%)$ \\
\hline GX $17+2$ & $90022-07-06-01$ & $19 / 09 / 2007$ & 09:11 & 2944.0 & 79.5 & 30.0 & 2 & 23.3 & 28.7 & 33.0 \\
\hline GX $17+2$ & $90022-07-07-00$ & $27 / 09 / 2007$ & $15: 15$ & 1392.0 & 168.1 & 53.7 & 3 & 17.5 & 19.6 & 21.3 \\
\hline Cyg X-2 & 93443-01-01-03 & $01 / 07 / 2008$ & $01: 34$ & 2016.0 & 644.3 & 40.8 & 2 & 6.8 & 8.3 & 9.4 \\
\hline Cyg X-2 & 93443-01-01-04 & $01 / 07 / 2008$ & $03: 12$ & 1776.0 & 602.1 & 37.0 & 2 & 5.8 & 6.7 & 7.6 \\
\hline Cyg X-2 & $93443-01-01-05$ & $01 / 07 / 2008$ & $04: 50$ & 1536.0 & 464.2 & 39.3 & 2 & 8.1 & 9.5 & 10.5 \\
\hline Cyg X-2 & 93443-01-01-06 & $01 / 07 / 2008$ & $06: 28$ & 1408.0 & 462.6 & 37.4 & 2 & 6.6 & 8.0 & 9.0 \\
\hline Cyg X-2 & 93443-01-01-08 & $01 / 07 / 2008$ & 11:09 & 1344.0 & 772.0 & 16.5 & 2 & 6.0 & 7.0 & 7.9 \\
\hline HETE J1900.1-2455 & $93030-01-16-00$ & $13 / 10 / 2007$ & $12: 49$ & 3392.0 & 88.3 & 55.2 & 3.0 & 27.8 & 34.9 & 40.8 \\
\hline HETE J1900.1-2455 & $93030-01-17-00$ & $21 / 10 / 2007$ & $10: 52$ & 3392.0 & 120.3 & 54.0 & 3.0 & 19.6 & 24.1 & 27.4 \\
\hline
\end{tabular}

Notes. $T_{\mathrm{obs}}$ is the observation duration (s) and rate and Bkg. give the source and background count rates (counts/s) respectively. The number of active PCA units $\left(N_{\mathrm{PCU}}\right)$ are listed for each Obs. ID. The upper limits on the QPO rms amplitude (\%) have been computed for QPO width of 50 , 100 , and $150 \mathrm{~Hz}$ (rms 50 , $\mathrm{rms}_{100}, \mathrm{rms}_{150}$, respectively). They have been computed at the $3 \sigma$ confidence level, meaning that the rms listed would enable the detection of any QPOs with a $3 \sigma$ accuracy on its amplitude (this corresponds to a $\sim 6 \sigma$ excess (single trial) in the PDS, see Boutelier et al. 2009 for details).

As we noted earlier, if both the upper $\mathrm{kHz} \mathrm{QPO}$ and $\mathrm{Fe}$ $\mathrm{K}$ emission line arise from the same region in the disk, and the $\mathrm{kHz} \mathrm{QPO}$ is associated with an orbital frequency, they can be combined to estimate the neutron star mass (Piraino et al. 2000; Cackett et al. 2008). If we tentatively use the inferred $910 \mathrm{~Hz}$ from scaling of the HBO frequency, along with the inner disk radius from the iron line we get a mass estimate in the range 1.5-2.4 $M_{\odot}$ (depending on whether we use the phenomenological or reflection modeling results), consistent with the optical mass measurement (Orosz \& Kuulkers 1999; Elebert et al. 2009). This implies a consistent radius between the $\mathrm{Fe} \mathrm{K}$ emission line and the $\mathrm{kHz}$ QPO. But, again, we are extremely cautious about this given the large uncertainty in scaling the QPO frequency, and the possible range of inner disk radii.

Looking at the RXTE archive, two observations of HETE J1900.1-2455 were performed contemporaneously with the Suzaku pointings of this source. Unfortunately, no $\mathrm{kHz}$ QPOs were detected. Upper limits are also listed in Table 9, but they are not very constraining on the presence of $\mathrm{kHz}$ QPOs due to the low source count rate and a comparatively large background.

Archival $R X T E$ observations are also available for the three $X M M-N e w t o n$ pointings of $4 \mathrm{U}$ 1636-53: the first observation was analyzed in Barret et al. (2005) and the latter two are also considered in Boutelier et al. (2010). We analyze the data using the same method as described above. As strong $\mathrm{kHz}$ QPOs were detected in many pointed observations, the QPO parameters (mainly the quality factor) were corrected for frequency drift using the method described in Boutelier et al. (2009). A total of 10 observations were considered. In four of these, we detect twin $\mathrm{kHz}$ QPOs, in one we detect a single upper $\mathrm{kHz}$ QPO at $\sim 500 \mathrm{~Hz}$ (i.e., the lower $\mathrm{kHz}$ QPO which is expected at much lower frequencies has merged with the noise and is no longer a QPO), and in two, a single lower $\mathrm{kHz}$ QPO (for an identification of single $\mathrm{kHz}$ QPO, based on their quality factor and rms amplitude see Barret et al. 2006). The non-detection of the upper $\mathrm{kHz}$ QPO in the latter two pointed observations is due to a lack of sensitivity (see Boutelier et al. 2010), but the frequency of the upper $\mathrm{kHz}$ QPO can be approximated by the nearly linear function that links the two frequencies (e.g., Belloni et al. 2005). The results of the fits are listed in Table 10.

These simultaneous XMM-Newton and RXTE observations are discussed extensively in Altamirano et al. (2010); here we briefly discuss the consistency of our spectral fitting with the $\mathrm{kHz}$ QPO properties. As Altamirano et al. (2010) find, when assuming a $1.4 M_{\odot}$ neutron star, the inner disk radius (as measured by the $\mathrm{Fe} \mathrm{K}$ line from phenomenological fits) disagrees with the implied inner disk radius assuming that the upper $\mathrm{kHz}$ QPO is a Keplerian frequency in the disk. If instead one uses the phenomenological $R_{\text {in }}$ measurements combined with the upper $\mathrm{kHz}$ QPO frequency to estimate a mass, the implied mass would be $4.2 \pm 0.5 M_{\odot}, 1.7 \pm 0.2 M_{\odot}$, and $2.2 \pm 0.1 M_{\odot}$ from the three observations, respectively (we use the $\mathrm{kHz}$ QPO frequencies from the RXTE observations that overlap with the XMM-Newton observations for the longest period). Clearly, the first observation estimates far too high a neutron star mass, while the other two observations give a more reasonable (though quite high) estimate. Note a high mass is predicted for 4U 1636-53 from the drop in $\mathrm{kHz}$ QPO coherence (Barret et al. 2006). From reflection modeling, we find slightly different $R_{\text {in }}$ measurements than for the phenomenological fits. The corresponding mass estimates from reflection fits are $2.6 \pm 1.0 M_{\odot}, 0.8 \pm 0.4 M_{\odot}$, and $0.7 \pm 0.2 M_{\odot}$. Again, the separate measurements are not all consistent with each other, and this time two of the masses are clearly on the low side.

Looking at the trends, if the two phenomena originate in the same part of the disk, a lower $\mathrm{kHz}$ QPO frequency should correspond to a larger inner disk radius (as measured by the $\mathrm{Fe} \mathrm{K}$ line). However, in 4U 1636-53 the opposite behavior is seen-as the $\mathrm{kHz}$ QPO frequency increases there is not a corresponding decrease in $R_{\text {in }}$, in fact, when using the $R_{\text {in }}$ measurements from reflection modeling $R_{\text {in }}$ is actually seen to increase. This, however, is only based on three observations, and further, higher quality observations are needed to make any strong conclusions.

\section{DISCUSSION}

We have presented a comprehensive study of $\mathrm{Fe} \mathrm{K}$ emission lines in 10 neutron star LMXBs. In all cases, the emission line profiles are modeled well by a relativistic disk line assuming a Schwarzschild metric. However, in one source, GX 340+0, the exact line profile is uncertain: a relativistic emission line fits the data well but only if either the abundance of Fe is non-standard or alternatively there is an Fe XXVI absorption line superposed on the emission line. Fitting of broadband spectra makes it clear that the hot blackbody component dominates the spectrum from around 7-20 keV (in the $\mathrm{Z}$ and atoll sources), and thus provides the vast majority of the flux irradiating the accretion disk and leading to the Fe $\mathrm{K}$ emission line. We fit reflection models to the spectra, finding that in the $\mathrm{Z}$ and atoll sources a reflection model 
Table 10

RXTE Observations of 4U 1636-536 Simultaneous with the Three XMM-Newton Observations

\begin{tabular}{|c|c|c|c|c|c|c|c|c|c|c|c|}
\hline Name & Obs. ID & Date & Time & $T_{\mathrm{obs}}$ & Rate & Bkg. & $N_{\mathrm{PCU}}$ & $v$ & FWHM & $\operatorname{rms}(\%)$ & $R$ \\
\hline $4 U 1636-53$ & 70034-01-01-01 & $9 / 08 / 2005$ & $3: 24$ & 4338.0 & 406.9 & 53.3 & 3 & $509.6 \pm 35.6$ & $191.4 \pm 85.8$ & $12.3 \pm 2.0$ & 3.0 \\
\hline $4 U$ 1636-53 & 91027-01-01-000 & $9 / 08 / 2005$ & $6: 35$ & 4434.0 & 0.6 & 78.8 & 3 & $\ldots$ & & & \\
\hline $4 U 1636-53$ & 91027-01-01-000 & $29 / 08 / 2005$ & $18: 06$ & 23362.0 & 47.4 & 78.9 & 3 & & & & \\
\hline $4 U 1636-53$ & 93091-01-01-000 & $28 / 09 / 2007$ & $14: 47$ & 4464.0 & 482.7 & 40.6 & 3 & $\begin{array}{l}643.5 \pm 2.5 \\
984.1 \pm 19.2\end{array}$ & $\begin{aligned} 17.8 & \pm 8.3 \\
165.3 & \pm 67.8\end{aligned}$ & $\begin{array}{r}6.5 \pm 1.0 \\
11.9 \pm 1.6\end{array}$ & $\begin{array}{l}3.4 \\
3.8\end{array}$ \\
\hline 4U 1636-53 & 93091- & $28 / 09 / 2007$ & $16: 17$ & 0 & 415.7 & 36.8 & 2 & $\begin{array}{l}589.7 \pm 10.5 \\
906.3 \pm 5.9\end{array}$ & $\begin{array}{l}94.6 \pm 31.8 \\
88.9 \pm 15.8\end{array}$ & $\begin{aligned} 7.8 & \pm 0.9 \\
10.0 & \pm 0.6\end{aligned}$ & $\begin{array}{l}4.2 \\
7.7\end{array}$ \\
\hline $4 \mathrm{U} 16$ & 93091 & $28 / 0$ & $22: 47$ & 7104.0 & 179.5 & 17.1 & 1 & $\ldots$ & $\ldots$ & $\ldots$ & $\ldots$ \\
\hline $4 \mathrm{U} 16$ & 9309 & $27 / C$ & $03: 46$ & 1280.0 & 690.2 & 58.3 & 5 & $645.9 \pm$ & $36.5 \pm 1$ & $8.4=$ & 3.4 \\
\hline 4U 1636-53 & 93091 & $27 / 02$ & $04: 35$ & 4352.0 & 688.6 & 34.9 & 3 & $\begin{array}{l}646.0 \pm 2.6 \\
979.5 \pm 27.1\end{array}$ & $\begin{aligned} 19.3 & \pm 7.4 \\
184.9 & \pm 71.8\end{aligned}$ & $\begin{array}{l}5.5 \pm 0.7 \\
9.3 \pm 1.3\end{array}$ & $\begin{array}{l}3.8 \\
3.6\end{array}$ \\
\hline 4U 1636-53 & 93091- & $27 / 02 / 2008$ & 06:07 & 20352.0 & 546.4 & 31.0 & 2 & $\begin{aligned} 701.6 & \pm 3.7 \\
1018.8 & \pm 32.8\end{aligned}$ & $\begin{aligned} 86.2 & \pm 13.5 \\
221.3 & \pm 111.4\end{aligned}$ & $\begin{array}{r}10.5 \pm 0.6 \\
7.8 \pm 1.4\end{array}$ & $\begin{array}{l}8.5 \\
2.8\end{array}$ \\
\hline $4 U 1636-53$ & 93091-01-02-00 & $27 / 02 / 2008$ & $11: 46$ & 10096.0 & 252.5 & 17.0 & 1 & $882.0 \pm 2.6$ & $15.8 \pm 5.8$ & $6.7 \pm 0.9$ & 3.7 \\
\hline
\end{tabular}

Notes. $T_{\mathrm{obs}}$ is the observation duration (s) and rate and Bkg. give the source and background count rates respectively. The number of active PCA units ( $\left.N_{\mathrm{PCU}}\right)$ are listed for each Obs. ID. The frequency, full width at half-maximum, and rms amplitude (\%) of each $\mathrm{kHz}$ QPO are reported with their $1 \sigma$ error. $R$ is the ratio of the Lorentzian amplitude to its $1 \sigma$ error. An $R$ of 3 corresponds roughly to $6 \sigma$ excess power in the Fourier PDS, see Boutelier et al. (2009) for details. When two QPOs are detected in one Obs. ID, the first one refers to the lower $\mathrm{kHz}$ QPO, whereas the second one refers to the upper $\mathrm{kHz}$ QPO.

where the irradiating source is a blackbody does fit the spectra well. In the accreting millisecond pulsars, however, a power law dominates above $10 \mathrm{keV}$, and standard reflection models used to fit black hole spectra work well there.

\subsection{Inner Disk Radii}

The inner disk radii that we measure mostly fall into a small range: $6-15 G M / c^{2}$. Also consistent with this range is a recent independent measurement of the inner disk radius in $4 \mathrm{U} 1705-44\left(R_{\text {in }}=14 \pm 2\right.$; Di Salvo et al. $\left.2009 \mathrm{c}\right)$. Where there are multiple observations of a source, in most cases there is not a large change in the inner disk radius, which may suggest that the inner disk radius does not vary with state. While we note this from observations at different epochs, similar results have been found previously when looking at how the line profile changes with source state during one observation (D'Aì et al. 2009; Iaria et al. 2009).

The only source where we see variations in inner disk radii is Ser X-1, and this is easily apparent when one compares the line profile observed by Suzaku with one of the XMM-Newton observations (see Figure 3). Comparing these observations, the spectrum from the Suzaku observation of Ser X-1 is harder than the XMM-Newton observations-although both the blackbody and disk blackbody components are hotter, the relative normalization of the blackbody component relative to the disk blackbody component is higher. The ionizing flux during the Suzaku observation is therefore stronger, which would explain the higher line flux. In all cases, though, the emitting radius inferred from the blackbody component is very similar. The $0.5-10 \mathrm{keV}$ source flux is also highest during the Suzaku observation $\left(10.3 \times 10^{-9} \mathrm{erg} \mathrm{cm}^{-2} \mathrm{~s}^{-1}\right.$ compared to $6.3 \times 10^{-9}, 4.4 \times 10^{-9}$, and $4.9 \times 10^{-9} \mathrm{erg} \mathrm{cm}^{-2} \mathrm{~s}^{-1}$ for the XMM-Newton observations). This may tentatively suggest that the disk extends closer to the stellar surface at the highest luminosities, though this is not confirmed when looking at all sources (Figure 7).

We have also looked to see if there is any significant change in the inner disk radius with $0.5-25 \mathrm{keV}$ luminosity for all sources (see Figure 7). There is no apparent systematic trend with luminosity, and the inner disk radius is found to be close to $6 G M / c^{2}$ for the majority of sources/observations over

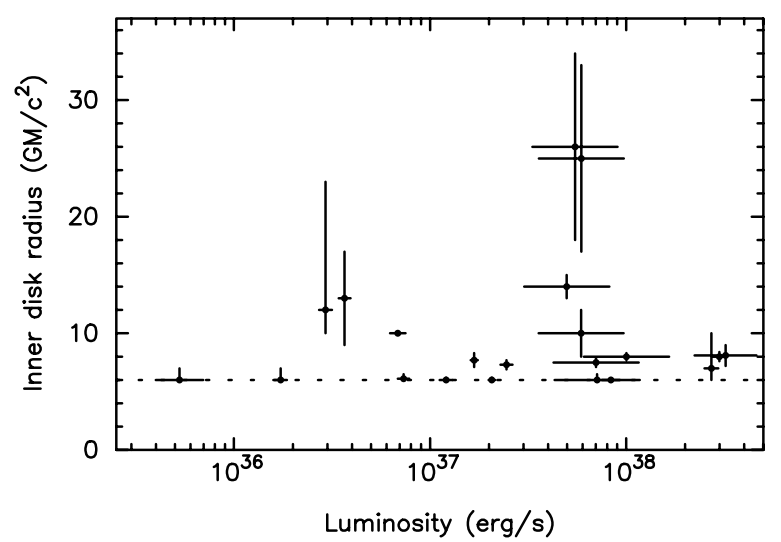

Figure 7. Measured inner disk radii from phenomenological fits as a function of $0.5-25 \mathrm{keV}$ source luminosity. The dotted line indicates $6 \mathrm{GM} / \mathrm{c}^{2}$, the smallest allowed radius in the model.

almost three orders of magnitude in luminosity. Similarly, we see no clear difference between atolls, $\mathrm{Z}$ sources, and accreting millisecond pulsars, as can be seen in Figure 4.

There is typically consistency between the inner disk radii measured from phenomenological and reflection spectral fits. Nevertheless, in both cases many of the inner disk radius measurements are pegged at the lower limit of the model (the ISCO). In 4U 1705-44, Reis et al. (2009b) found that fitting with a model for a Kerr metric (relevant for maximally spinning black holes; Laor 1991) gave inner disk radii much smaller than the expected neutron star radii. They suggested that as the disks in these systems are ionized one must also take broadening due to Comptonization into account by using relativistic ionized reflection models. When they did this, the inner radius measured was significantly higher, and no longer smaller than the expected stellar radii. The reflection models employed here also take Compton broadening into account, yet, we still find that the inner disk radius pegs at the ISCO in a number of sources.

This may not necessarily be a problem. As neutron stars are spinning, their angular momentum will change the radius of the ISCO making it slightly smaller for a corotating disk (see Figure 1(a) of Miller 2007 to see how the ISCO changes with angular momentum). Potentially, one could then measure 
Table 11

Disk Blackbody Inner Radius

\begin{tabular}{|c|c|c|c|c|c|c|c|c|}
\hline \multirow[t]{2}{*}{ Source } & \multirow[t]{2}{*}{ Mission } & \multirow[t]{2}{*}{ Obs. ID } & \multicolumn{3}{|c|}{ Phenomenological } & \multicolumn{3}{|c|}{ Reflection } \\
\hline & & & $R_{\text {inner }}(\mathrm{km})$ & $\begin{array}{c}R_{\text {in }}(G M / c 2) \\
\text { for } 1.4 M_{\odot}\end{array}$ & $\begin{array}{c}R_{\text {in }}\left(G M / c^{2}\right) \\
\quad \text { for } 2 M_{\odot}\end{array}$ & $R_{\text {inner }}(\mathrm{km})$ & $\begin{array}{c}R_{\text {in }}\left(G M / c^{2}\right) \\
\text { for } 1.4 M_{\odot}\end{array}$ & $\begin{array}{l}R_{\text {in }}\left(G M / c^{2}\right) \\
\quad \text { for } 2 M_{\odot}\end{array}$ \\
\hline \multirow[t]{4}{*}{ Serpens X-1 } & Suzaku & 401048010 & $8.7 \pm 2.2$ & $4.2 \pm 1.0$ & $2.9 \pm 0.7$ & $9.0 \pm 2.3$ & $4.4 \pm 1.1$ & $3.0 \pm 0.8$ \\
\hline & XMM-Newton & 0084020401 & $13.1 \pm 3.2$ & $6.3 \pm 1.6$ & $4.4 \pm 1.1$ & $11.7 \pm 2.9$ & $5.6 \pm 1.4$ & $4.0 \pm 1.0$ \\
\hline & XMM-Newton & 0084020501 & $11.3 \pm 2.8$ & $5.5 \pm 1.4$ & $3.8 \pm 1.0$ & $10.9 \pm 2.7$ & $5.3 \pm 1.3$ & $3.7 \pm 0.9$ \\
\hline & XMM-Newton & 0084020601 & $12.7 \pm 3.2$ & $6.1 \pm 1.5$ & $4.3 \pm 1.1$ & $11.3 \pm 2.8$ & $5.5 \pm 1.4$ & $3.8 \pm 1.0$ \\
\hline \multirow[t]{3}{*}{$4 U 1636-53$} & XMM-Newton & 0303250201 & $143 \pm 72$ & $70 \pm 35$ & $49 \pm 25$ & $100 \pm 49$ & $49 \pm 24$ & $34 \pm 17$ \\
\hline & XMM-Newton & 0500350301 & $12.7 \pm 6.3$ & $6.2 \pm 3.0$ & $4.3 \pm 2.1$ & $7.9 \pm 2.2$ & $3.8 \pm 1.1$ & $2.7 \pm 0.7$ \\
\hline & XMM-Newton & 0500350401 & $18.8 \pm 9.4$ & $9.1 \pm 4.6$ & $6.4 \pm 3.2$ & $10.8 \pm 5.3$ & $5.2 \pm 2.6$ & $3.7 \pm 1.8$ \\
\hline \multirow[t]{4}{*}{$4 \mathrm{U} 1705-44$} & Suzaku & 401046010 & $2910_{-1020}^{+4560}$ & $1400_{-490}^{+2200}$ & $990_{-340}^{+1540}$ & $9780_{-3590}^{+6410}$ & $4740_{-1740}^{+3100}$ & $3320_{-1220}^{+2170}$ \\
\hline & Suzaku & 401046020 & $4.5 \pm 0.2$ & $2.2 \pm 0.1$ & $1.5 \pm 0.1$ & $6.5 \pm 0.3$ & $3.2 \pm 0.1$ & $2.2 \pm 0.1$ \\
\hline & Suzaku & 401046030 & $5.3 \pm 0.2$ & $2.6 \pm 0.1$ & $1.8 \pm 0.1$ & $5.5 \pm 0.4$ & $2.7 \pm 0.2$ & $1.9 \pm 0.1$ \\
\hline & XMM-Newton & 0402300201 & $1.7 \pm 0.9$ & $0.8 \pm 0.4$ & $0.6 \pm 0.3$ & $0.96 \pm 0.04$ & $0.47 \pm 0.02$ & $0.33 \pm 0.01$ \\
\hline $4 U$ 1820-30 & Suzaku & 401047010 & $7.8 \pm 0.5$ & $3.8 \pm 0.2$ & $2.6 \pm 0.2$ & $8.8 \pm 0.5$ & $4.3 \pm 0.2$ & $3.0 \pm 0.2$ \\
\hline \multirow[t]{2}{*}{ GX $17+2$} & Suzaku & 402050010 & $9.2 \pm 0.6$ & $4.5 \pm 0.3$ & $3.1 \pm 0.2$ & $9.8 \pm 0.5$ & $4.8 \pm 0.2$ & $3.3 \pm 0.2$ \\
\hline & Suzaku & 402050020 & $10.0 \pm 0.5$ & $4.8 \pm 0.2$ & $3.4 \pm 0.2$ & $9.6 \pm 0.4$ & $4.6 \pm 0.2$ & $3.2 \pm 0.2$ \\
\hline \multirow[t]{3}{*}{ GX $349+2$} & Suzaku & 400003010 & $5.6 \pm 1.4$ & $2.7 \pm 0.7$ & $1.9 \pm 0.5$ & $5.6 \pm 1.4$ & $2.7 \pm 0.7$ & $1.9 \pm 0.5$ \\
\hline & Suzaku & 400003020 & $5.9 \pm 1.5$ & $2.8 \pm 0.7$ & $2.0 \pm 0.5$ & $5.4 \pm 1.4$ & $2.6 \pm 0.7$ & $1.8 \pm 0.5$ \\
\hline & XMM-Newton & 0506110101 & $10.6 \pm 2.7$ & $5.1 \pm 1.3$ & $3.6 \pm 0.9$ & $9.1 \pm 2.3$ & $4.4 \pm 1.1$ & $3.1 \pm 0.8$ \\
\hline Cyg X-2 & Suzaku & 403063010 & $14.8 \pm 2.7$ & $7.2 \pm 1.3$ & $5.0 \pm 0.9$ & $14.1 \pm 2.6$ & $6.8 \pm 1.2$ & $4.8 \pm 0.9$ \\
\hline \multirow[t]{2}{*}{ SAX J1808 } & Suzaku & 903003010 & $1.1 \pm 0.1$ & $0.51 \pm 0.04$ & $0.36 \pm 0.03$ & $20.7 \pm 2.0$ & $10.0 \pm 1.0$ & $7.0 \pm 0.7$ \\
\hline & XMM-Newton & 0560180601 & $70_{-10}^{+23}$ & $34_{-5}^{+11}$ & $24_{-3}^{+8}$ & $8.5 \pm 0.5$ & $4.1 \pm 0.2$ & $2.9 \pm 0.2$ \\
\hline
\end{tabular}

Note. Assumed distances are given in Table 1.

the dimensionless angular momentum parameter, under the assumption that the inner disk is truncated at the ISCO and not at the boundary layer or stellar surface. To test this, we fit a line profile with a variable angular momentum parameter (Brenneman \& Reynolds 2006) to several of the best data sets in our sample. However, in each case we found that this parameter was not well constrained, being consistent with zero. Better, more sensitive data may be able to constrain this further.

We can also compare the inner disk radii from the Fe line fitting with that implied from the disk blackbody fits. The normalization of the disk blackbody used here (diskbb) is defined as $N=R_{\text {inner }}^{2} \cos i /(D 10)^{2}$, where $R_{\text {inner }}$ is the inner disk radius in $\mathrm{km}, D 10$ is the source distance in units of $10 \mathrm{kpc}$, and $i$ is the disk inclination. Therefore, from the fitted normalization and the inclination determined from the Fe line fits, we can calculate the inner disk radius in kilometers. Assuming a reasonable range for the neutron star mass (here we use 1.4-2.0 $M_{\odot}$ ) we can convert to units of $G M / c^{2}$ for direct comparison with the $\mathrm{Fe}$ line fits. The disk radii determined this way are given in Table 11. Where only an lower/upper limit for the inclination is found from the Fe line fits, we just use this limit for the inclination in the calculation.

Comparing the inner disk radii from the continuum (diskbb) and $\mathrm{Fe}$ line fitting, we find that the continuum inner disk radius is generally smaller than the Fe line value, though there are a small number of cases where the values are consistent (when assuming a mass of $1.4 M_{\odot}$ ). It is important to note that we have not used any spectral hardness factor or a correction for the inner boundary condition for the continuum radii. These two factors are expected to lead to a factor of 1.18-1.64 increase between the radius measured by diskbb and the real inner disk radius (e.g., Kubota et al. 2001). This would bring more of the values to be consistent, though a large number would still disagree. Where there are large disagreements, it could be possible that the continuum model chosen is not physical. For disagreements where the continuum radius is smaller than the Fe line radius, one can imagine that disk photons may be scattered out of the line of sight by a corona, diminishing the strength of the corona, and leading to a smaller measured continuum radius. Additionally, the continuum method relies on knowing the absolute flux of the disk component. Therefore, if the absolute flux calibration of the telescope or the continuum model (column density or other parameters) is incorrect, then this can lead to an incorrect inner disk radius.

\subsection{Source Inclination and Disk Emissivity}

We found in many cases that there are several local $\chi^{2}$ minima within the diskline parameter space. There is usually one solution that is clearly the global minimum, with a significantly better $\chi^{2}$ value. However, we found that in one instance (GX $349+2$, second $S u z a k u$ observation) the difference was as small as $\Delta \chi^{2} \sim 2$. This issue is due to a degeneracy where a model with a combination of a low-inclination, high emissivity index and high line energy can look similar to a higher inclination, lower emissivity index and a lower line energy model. Usually the inner disk radius remains consistent between the solutions. This is apparent in Table 5, see particularly the GX 349+2 and Ser X-1 observations. We demonstrate this issue by showing the dependence of the $\chi^{2}$ confidence contours on inclination and emissivity index for the second GX 349+2 Suzaku observation in Figure 8 . Here, one clearly sees two minima with only a small difference in $\chi^{2}$. The $\chi^{2}$ minimum is 1262.6 for the low emissivity index, high inclination minimum compared to 1265.0 for the high emissivity index, low inclination local minimum (both have 1106 dof). For the first Suzaku GX 349+2 we also find two minima. In this case, however, the global minimum has a high emissivity index, low inclination opposite to the second observation $\left(\chi^{2}=1268.1\right.$ for low inclination compared to 1273.6 for higher inclination).

As discussed, comparing all observations of GX 349+2, the inclination and emissivity index from spectral fitting varies significantly (see Table 5). This was first pointed out by Iaria 


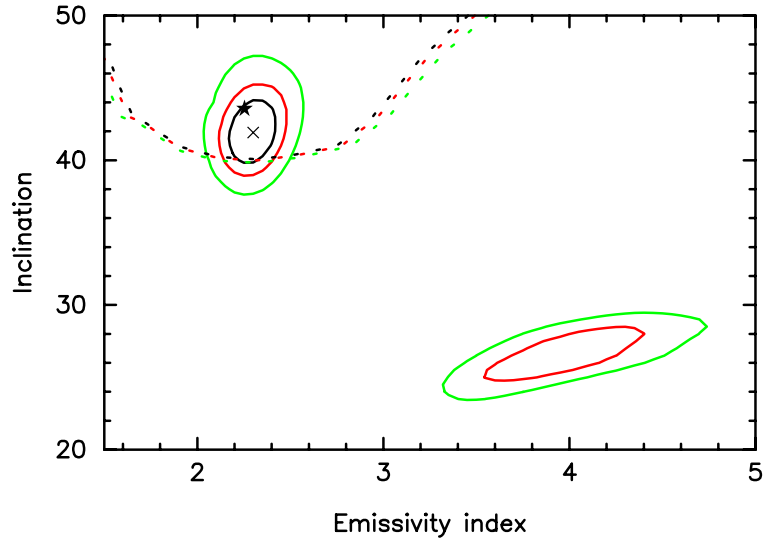

Figure 8. Dependence of the $\chi^{2}$ confidence contours on inclination and emissivity index for GX $349+2$. The cross marks the global $\chi^{2}$ minimum when the second Suzaku observation is fit on its own, and the star marks the minimum when all three observations of GX $349+2$ are fit jointly, with the inclination tied between them. The contours are the $1 \sigma$ (black), $90 \%$ (red), and 99\% (green) confidence levels, with the solid lines from fitting the second observation on its own, and the dotted lines from when all three observations are fit jointly.

et al. (2009) when comparing their XMM-Newton results with the Cackett et al. (2008) results. As they also noted, while it is possible that the emissivity index, inner disk radius, and the line energy may vary with source state, the source inclination obviously does not. As we have demonstrated, this difference is not because the lines look very different. In fact, if all three observations of GX 349+2 are fit simultaneously with the inclination tied between observations (best fit value $i=44_{-4}^{+11}$ $\mathrm{deg}$ ), then a good fit is achieved, and the lines look almost identical (see Figure 9). Plainly, a priori knowledge of source inclination is of significant benefit. However, joint fitting of multiple observations of the same source potentially resolves this issue. As can been seen in Figure 8 when the three observations of GX 349+2 are fit jointly, there is no longer a local $\chi^{2}$ minimum with a high emissivity index and low inclination.

Such a degeneracy between the line parameters is self-evident when studying how each parameter affects the line profile (see Figure 1 of Fabian et al. 1989). The inclination shifts the peak of the blue wing of the line profile. Therefore, a higher line energy can be offset by a lower inclination. Moreover, a higher inclination makes the line broader and less "peaked," and the emissivity index also has a similar effect. Thus, a higher inclination and lower emissivity index can look similar to a lower inclination and higher emissivity index.

We find a similar problem with the inclination determined from fitting the Cyg X-2 spectra, where we measure an inclination of $25^{\circ}$. This inclination is not consistent with the value measured from optical observations, where lower and upper inclination limits are $49^{\circ}-73^{\circ}$, with a best-fitting value of $63^{\circ}$ (Orosz \& Kuulkers 1999; Elebert et al. 2009). If we constrain the inclination to be within the optical values, we do not achieve as good a fit $\left(\chi^{2}=1337\right.$ compared to $\left.\chi^{2}=1305\right)$, and find the inclination pegs at the lower bound of $49^{\circ}$. With the inclination constrained, the line parameters we find are $E=6.40^{+0.01} \mathrm{keV}$, $q=2.9 \pm 0.2, R_{\mathrm{in}}=25 \pm 4 G M / c^{2}, i=49^{+1 \circ}$. Again, there is a pattern where a higher inclination leads to a lower line energy and emissivity index.

Note that in several cases we find high emissivity indices and/or very low inclinations when fitting the reflection models. Both a high emissivity index and a low inclination try to make the line profile more peaked. We tested whether this can be

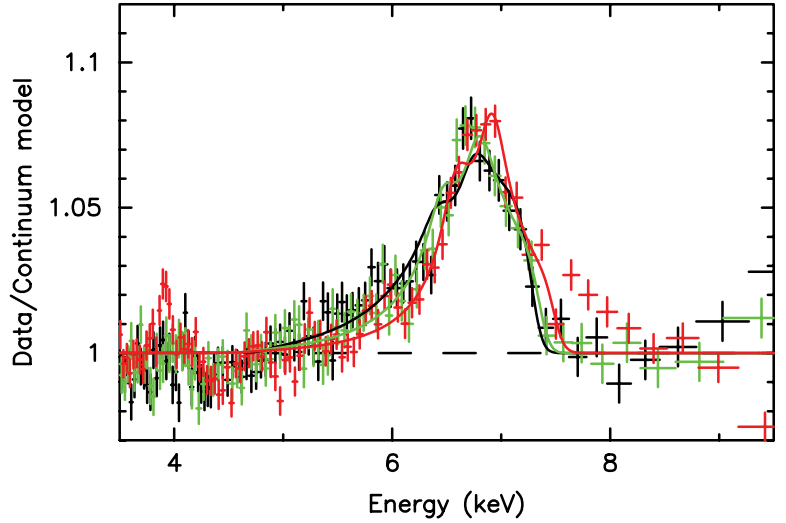

Figure 9. Fe K line profile (shown as data/continuum model) for the three observations of GX 349+2. Here the inclination was tied between the observations during the spectral fitting. Black shows the first Suzaku observation, green the second, and red the only XMM-Newton observation.

solved with a higher Fe abundance by using a reflection model with two times solar abundance, but retrieved similar results, and worse fits. Light bending can potentially lead to a high emissivity index, causing the irradiation of the disk to be more centrally concentrated. In the most extreme case of spinning black holes an index of $\sim 5$ is expected (Miniutti et al. 2003). These effects should not be as strong in neutron stars, and thus a lower index would be expected.

It is also important to note that in the reflection modeling the inclination is only introduced by the relativistic blurring, and the reflection spectrum itself is angle averaged. However, reflection is sensitive to angle, with the reflection strength weakening with higher inclination. The use of an angle-averaged model may therefore bias the results obtained.

In black hole systems, the ionizing flux is dominated by a power law. The Compton back-scattering part of the reflection spectrum is easily detected against a power-law continuum. Disk reflection is more difficult to detect against a thermal spectrum since the continuum flux drops precipitously with higher energy. In the case of neutron stars, then, disk reflection modeling may only yield improved physical constraints in the limit of extraordinary high energy sensitivity. The fact that many of our reflection fits imply small radii and low inclinations may be partially due to a combination of modest high energy sensitivity, coupled with the limitations of angle-averaged disk reflection models. Deeper observations with Suzaku aimed at clearly detecting the Compton back-scattering hump will help to obtain better physical constraints.

\subsection{Ionization Parameter and Reflection Fraction}

Reflection modeling allows us to infer the ionization state of the inner accretion disk. The reflection spectrum is highly dependent on the ionization parameter, $\xi$ (see Figure 1 of Ballantyne 2004). For low ionization states many emission lines are present which disappear as the ions become more highly ionized. The $\mathrm{Fe} \mathrm{K} \alpha$ line, in particular, evolves significantly with the ionization parameter. For neutral reflection, the $6.4 \mathrm{keV}$ line is strong and as the ionization increases this line becomes weaker. The $6.7 \mathrm{keV}$ line then becomes prominent until it disappears as iron becomes fully ionized. This evolution is displayed clearly in Figure 3 of Ballantyne (2004), where the dependence of the $\mathrm{EW}$ of the $\mathrm{Fe} \mathrm{K} \alpha$ on the ionization parameter is shown. The EW peaks either for neutral reflection when the $6.4 \mathrm{keV}$ line is strong or for $\log \xi=2.6-2.8$ when the $6.7 \mathrm{keV}$ 
line is dominant. From our reflection fitting (Tables 7 and 8) we find quite a narrow range of ionization parameters. Most spectra are best fit with an ionization parameter in the range $\log \xi=2.6-2.8$, where the Fe K $\alpha$ EW peaks.

We also consider the strength of the reflection component, which directly relates to the solid angle subtended by the reflector, $\Omega$. The reflection fraction, $R=\Omega / 2 \pi$, is given in Table 8 . A reflection fraction of 1 corresponds to an isotropic source above the slab. Most of the observations here have a reflection fraction in the range $R=0.1-0.3$, suggesting that a reasonable fraction of the ionizing flux is intercepted by the accretion disk and re-emitted. If we interpret the blackbody component as the boundary layer, this may also suggest that the boundary layer has quite a similar extent in these sources. As discussed above, Figure 3 of Ballantyne (2004) shows a strong dependence of the $\mathrm{Fe} \mathrm{K} \alpha$ line $\mathrm{EW}$ and the blackbody temperature. Most of the blackbody temperatures we find have $k T>2 \mathrm{keV}$, which equates to an $\mathrm{EW}$ of $>200 \mathrm{eV}$ for $R=1$. From the phenomenological fits (see Table 5), we get EWs that are mostly less than $100 \mathrm{eV}$; thus, the reflection fractions that we find generally seem reasonable.

There are two cases (one spectra of 4U 1705-44 and one spectra of $4 \mathrm{U} 1636-53)$ where the best-fitting reflection fractions are at the upper limit of the model $(R=5)$. In these spectra, either the blackbody temperature or the blackbody normalization is very low. Both these lead to a low EW, and therefore for the model to achieve the required line strength a large reflection fraction is required. Both these sources are atolls which do transit between hard and soft states (see, e.g., Gladstone et al. 2007), and these two particular observations are in the hard state. As discussed earlier, spectral deconvolution in atoll and $\mathrm{Z}$ sources is not unique (e.g., Lin et al. 2007), and the high values of the reflection fraction suggest that our particular choice of the model here is unlikely the best physical model. We have also tried replacing the blackbody reflection model with a power-law reflection model. While this achieved more reasonable reflection fractions it did not provide as good a fit to the data. D'Aì et al. (2010) have recently successfully modeled the hard state in 4U 1705-44 using an alternative model involving a Comptonization model to fit the hard component, and such a model may be more appropriate for these two particular observations, though we do not investigate this here.

\subsection{The Boundary Layer Illuminating the Disk}

It is well known that a hot blackbody (or alternatively a Comptonized component) dominates from around 7-20 keV (e.g., Barret et al. 2000). In all sources but the accreting millisecond pulsars, our spectral fitting agrees with this. This blackbody component may potentially be due to emission from the boundary layer between the inner accretion disk and the neutron star surface (see, e.g., Sunyaev \& Shakura 1986; Inogamov \& Sunyaev 1999; Popham \& Sunyaev 2001; Grebenev \& Sunyaev 2002; Gilfanov et al. 2003; Revnivtsev \& Gilfanov 2006, for discussions about the boundary layer). The boundary layer is the transition region between the rapidly spinning accretion disk and the more slowly spinning neutron star, and should account for a significant fraction of the accretion luminosity.

As neutron star spectra show that the blackbody component dominates the flux able to ionize iron, it suggests that the boundary layer may be the dominant source of ionizing flux illuminating the inner accretion disk, leading to $\mathrm{Fe} \mathrm{K} \alpha$ emission.
Our spectral analysis with reflection models shows that this picture is consistent with the data.

It is not a new idea that the boundary layer or neutron star surface can illuminate the disk leading to Fe $\mathrm{K}$ emission. For instance, Day \& Done (1991) were the first to note that X-ray bursts on the surface of the neutron star can lead to a diskreflection component. Furthermore, Brandt \& Matt (1994) use a geometry where an inner spherical cloud of Comptonized gas acts to illuminate the inner accretion disk as a basis for calculating $\mathrm{Fe} \mathrm{K}$ profiles expected in $\mathrm{Z}$ sources. Moreover, detailed calculations of the inner disk and boundary layer led Popham \& Sunyaev (2001) to conclude that the X-ray flux from the hot boundary layer incident on the accretion disk would be large enough to lead to line emission and a reflection spectrum.

Observationally, there is not a large body of previous work fitting broadband neutron star spectra with reflection models where the boundary layer irradiates the accretion disk. However, Done et al. (2002) successfully fit Ginga data of Cyg X-2 with a reflection model that illuminates the disk with a Comptonized continuum, and similarly Gierliński \& Done (2002) achieved good fits to the RXTE spectra of atoll 4U 1608-52 with the same model. However, the data in both cases had significantly lower spectral resolution to those presented here. Di Salvo et al. (2000a) also successfully fit the broadband BeppoSAX spectrum of MXB 1728-34 with a reflection model which illuminates the disk with a Comptonized continuum. Furthermore, Barret et al. (2000) suggest that the boundary layer provides the X-ray flux irradiating the disk from spectral fitting of $R X T E$ data.

The Popham \& Sunyaev (2001) model for the boundary layer is the one where the hot boundary layer gas is radially and vertically extended. Popham \& Sunyaev (2001) showed that the hot boundary layer is much thicker than the inner disk (see their Figure 1). As mass accretion rate changes they find that both the radial and vertical extent of the boundary layer will change, and thus the fraction of X-ray emission from the boundary layer that is intercepted by the disk will vary. An alternative model for the boundary layer is one where matter spreads over the neutron star surface in a latitudinal belt (Inogamov \& Sunyaev 1999). In this spreading layer, the belt width is dependent on the mass accretion rate. At low mass accretion rates $\left(3 \times 10^{-3}\right)$ the width is of the order of the thin-disk thickness; however, the belt disappears at high Eddington fractions $(\sim 0.9)$ as the entire surface is radiating. It therefore seems reasonable that even the spreading layer may plausibly illuminate the inner accretion disk.

We now further investigate the notion that the boundary layer is the source of ionizing flux. Using ionization parameters typical to what we find from spectral fitting, we determine the maximum height of the boundary layer, for a disk extending close to the neutron star surface. The ionization parameter at the inner accretion disk, $\xi$, is given by

$$
\xi=\frac{L_{\mathrm{BL}}}{n R^{2}},
$$

where $L_{\mathrm{BL}}$ is the boundary layer luminosity, $n$ is the number density in the disk, and $R$ is the distance from the ionizing source to the disk. For the purposes of this estimation we use the distance between the top of the boundary layer and the inner accretion disk. That distance is given by

$$
R=\sqrt{R_{\mathrm{in}}^{2}+Z^{2}},
$$

where $R_{\text {in }}$ is the inner disk radius, and $Z$ is the height of the ionizing source above the disk. The distance will, of course, be 
Table 12

Blackbody Emitting Radius

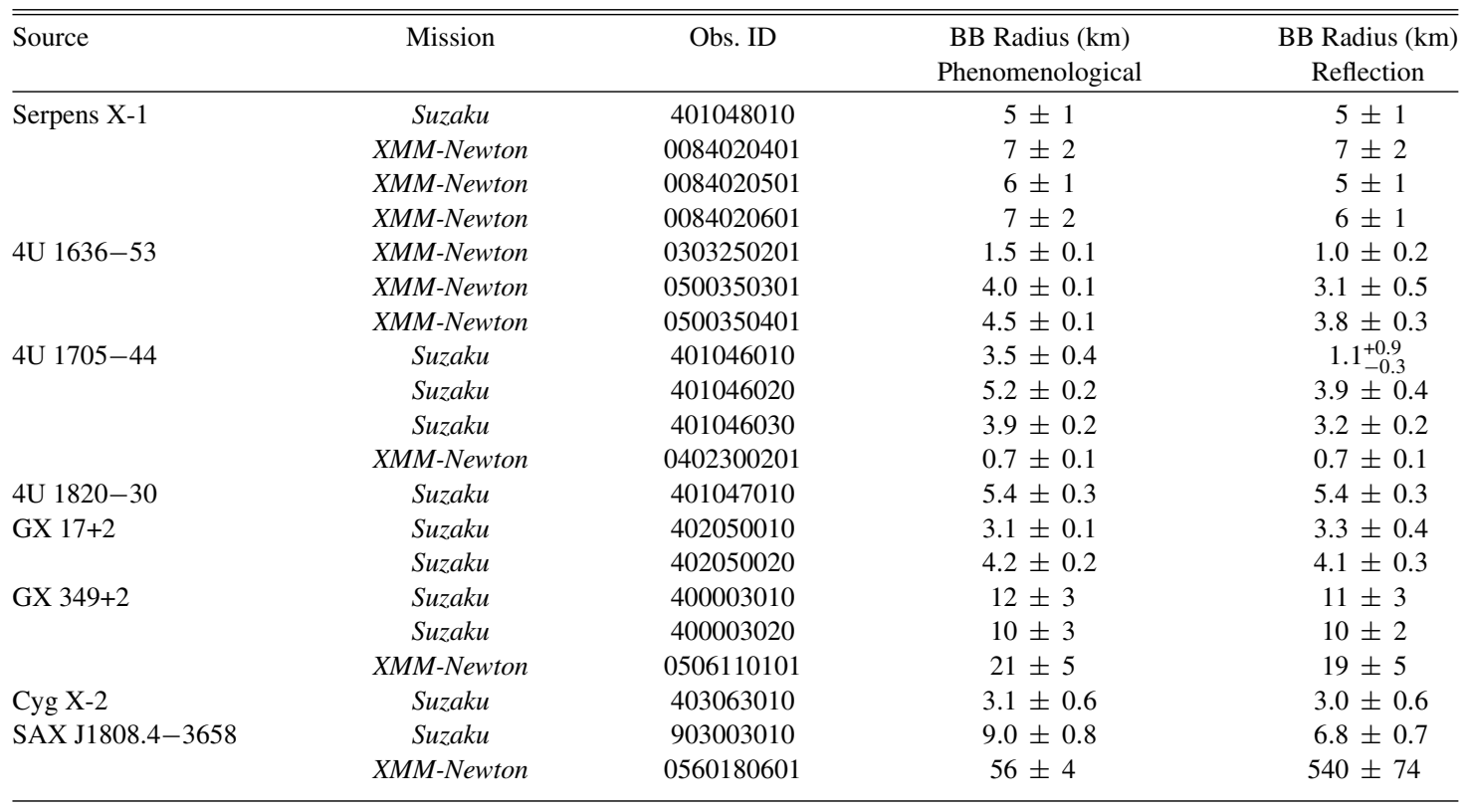

Note. Assumed distances are given in Table 1.

higher for other regions of the accretion disk, and for boundary layer emission closer to the equator. However, combining these two equations and re-arranging leads to the height of the ionizing source above the disk being defined as

$$
Z^{2}=\frac{L_{\mathrm{BL}}}{n \xi}-R_{\mathrm{in}}^{2} .
$$

From spectral fitting and reflection modeling we determine $L_{\mathrm{BL}}$, $R_{\text {in, }}$ and $\xi$, and from standard disk theory we can put reasonable constraints on $n$. From Shakura \& Sunyaev (1973), we find $n>10^{21} \mathrm{~cm}^{-3}$. For $\log \xi=3, L_{\mathrm{BL}}=10^{37} \mathrm{erg} \mathrm{s}^{-1}$ and $R_{\text {in }}=20 \mathrm{~km}$, we find $Z<24 \mathrm{~km}$. It is therefore completely reasonable that the boundary layer provides the ionizing flux to produce the $\mathrm{Fe} \mathrm{K}$ emission lines observed. A subsequent detailed analysis of multiple spectra from 4U 1705-44 has also concluded that the boundary layer likely illuminates the disk, at least in high flux states (D'Aì et al. 2010).

A further check can be performed by looking at the inferred blackbody emitting radius from the spectral fits. In Table 12, we show the calculated emitting radius and assumed distances from the blackbody normalizations. We use the same distances as given in Table 11. As the boundary layer emission is more correctly described by a Comptonized component as opposed to a simple blackbody we can maybe not interpret these numbers directly. However, they do provide a good consistency check, with radii typically being a significant fraction of the stellar radius, as expected theoretically (Popham \& Sunyaev 2001). In a couple of cases the radii are extremely high, in those few cases it seems likely that while the model may be a good fit to the data it is not physically correct.

In many cases, we find that the hot blackbody component contributes substantially to the overall flux. Substantial boundary layer emission is consistent with observed spins in neutron stars in LMXBs, which spin slower than the Keplerian angular speed in the inner accretion disk. If a neutron star had significantly higher angular speed, the boundary layer emission is expected to tend to zero as the angular speed approached the Keplerian angular speed (see, for example, Figure 5 from Bhattacharyya et al. 2000).

\subsection{Alternative Emission Line Model}

We interpret the observed $\mathrm{Fe} \mathrm{K}$ emission line as originating in the inner accretion disk, with relativistic effects giving rise to the asymmetric line profile (as is generally accepted). There has, however, been a suggestion that these asymmetric Fe K lines can originate from an optically thick high-velocity flow in these systems (Titarchuk et al. 2003; Laming \& Titarchuk 2004; Laurent \& Titarchuk 2007; Shaposhnikov et al. 2009; Titarchuk et al. 2009). In this model, a diverging wind with a wide opening angle is launched from the disk. The Fe $\mathrm{K}$ line is formed in a narrow wind shell that is illuminated by X-rays from the accretion disk. The asymmetric profile arises when $\mathrm{Fe} \mathrm{K}$ photons scatter off electrons in the partly ionized wind, becoming redshifted in the process. These photons undergo a large number of multiple scatterings to form the red wing of the line. Such a model appears to fit some Fe K line profiles well (Shaposhnikov et al. 2009; Titarchuk et al. 2009).

To reproduce the observed line profiles requires that there be a very large number of electron scatters in the wind, and hence the wind had a high optical depth (Titarchuk et al. 2009 find $\tau_{\text {wind }}=1.6$ for neutron star LMXB Ser X-1 and $\tau_{\text {wind }}=4.9$ for black hole X-ray binary GX 339-4). The mass outflow rate required for GX 339-4 is comparable to the Eddington mass inflow rate. In the low/hard state considered by Titarchuk et al. (2009), this means that the outflow rate is $30-100$ times higher than the inferred mass inflow rate. The outflow rate required to produce the line in Ser X-1 is also comparable to the Eddington mass inflow rate. Not only are high outflow rates required, but the outflow must also not be coupled to the inflow rate given that we see similar line profiles in the neutron star systems over more than two orders of magnitude in luminosity.

Wide-angle, optically thick outflows should have observable consequences in X-rays and in other bands. In X-rays, ionizing flux from the central engine should generate absorption lines 


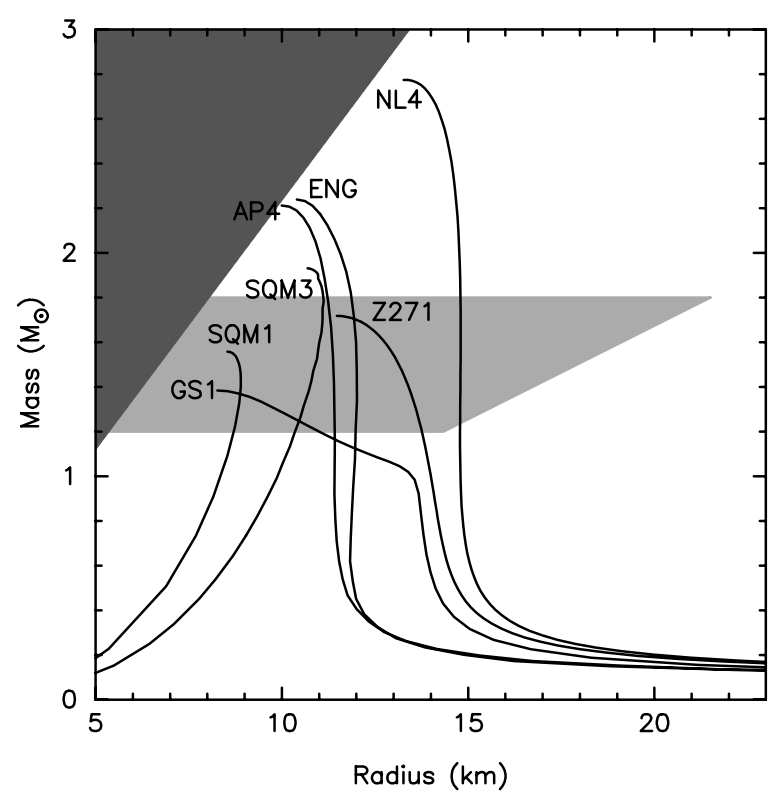

Figure 10. Constraints on the ultra-dense matter equation of state from the mass and radius upper limit of Cyg X-2 (light gray region). The dark gray region is not allowed due to causality. All mass-radius curves are as labeled in Lattimer \& Prakash (2001; see references therein for details of the equations of state), except for NL4 which is from Akmal et al. (1998) and Z271 which is from Horowitz \& Piekarewicz (2001).

for any line of sight through the wind. Yet when absorption lines are observed in black holes, they are always slow, with velocities less than approximately $1000 \mathrm{~km} \mathrm{~s}^{-1}$ (see, e.g., Miller et al. 2006b, 2008a on GRO J1655-40 and Ueda et al. 2009 on GRS $1915+105)$. In some cases, absorption lines are seen in the absence of any relativistic line (e.g., H 1743-322 and 4U 1630-472; Miller et al. 2006c; Kubota et al. 2007). The absorption lines detected in most neutron star systems (the edgeon "dipping" sources) are often consistent with no velocity shift (e.g., Sidoli et al. 2001; Parmar et al. 2002; Boirin et al. 2005; Díaz Trigo et al. 2006). Clearly, observed absorption spectra are in strong disagreement with the wind model.

In optical and IR bands, optically thick diverging outflows should also have consequences. Whether in a black hole, neutron star, or white dwarf system, the outflow should serve to obscure our view of the outer accretion disk and/or companion star, at least during some intervals of the binary period. Along particular lines of sight, the outer disk should be obscured for nearly all of the binary period. Obscuration of this kind has not been reported, though it would surely be easily detected. Doppler tomography reveals no evidence of such outflows as accretion disk, stream/ hot spot, and companion star emission are often clearly revealed (e.g., Marsh et al. 1994; Steeghs \& Casares 2002).

Finally, it is worth noting that relativistic lines are not detected in white dwarf systems. Titarchuk et al. (2009) fit their model to the XMM-Newton CCD spectrum of GK Per, where they only observe one emission line. This, however, ignores the fact that high-resolution Chandra grating observations clearly separated the emission into three separate $\mathrm{Fe}$ components, as is seen in many magnetic cataclysmic variables (CVs; Hellier \& Mukai 2004). The spectra of CVs are best described in terms of partially obscured emission from the boundary layer, which can be fitted with cooling-flow models that predict multiple iron charge states (Hellier \& Mukai 2004).

\subsection{Equation of State}

To begin to put meaningful constraints on the neutron star equation of state, it is useful to combine both mass and radius measurements. Of the sources in our sample, Cyg X2 is the only one with a well-constrained mass measurement, $M_{\mathrm{NS}}=1.5 \pm 0.3 M_{\odot}$ (Elebert et al. 2009). Combining this mass measurement with our stellar radius upper limit $\left(8.1 \mathrm{GM} / \mathrm{c}^{2}\right)$ can be seen in Figure 10. This does not rule out any of the possible equations of state shown here, and tighter constraints on mass and radius would be needed.

Significantly improved sensitivity and spectral resolution from future X-ray missions, such as Astro- $H$ and the International $X$-ray Observatory (IXO), will certainly provide tighter constraints from modeling Fe $\mathrm{K}$ emission lines, and with the High Timing Resolution Spectrometer on IXO even the potential to study simultaneous spectral and timing evolution on extremely short timescales (Barret et al. 2008).

We appreciate the extremely helpful comments from the referee that have improved the paper. E.M.C. gratefully acknowledges support provided by NASA through the Chandra Fellowship Program, grant number PF8-90052. This research has made use of data obtained from the Suzaku satellite, a collaborative mission between the space agencies of Japan (JAXA) and the USA (NASA). It also made use of observations obtained with XMM-Newton, an ESA science mission with instruments and contributions directly funded by ESA Member States and NASA.

\section{REFERENCES}

Akmal, A., Pandharipande, V. R., \& Ravenhall, D. G. 1998, Phys. Rev. C, 58, 1804

Altamirano, D., Hiemstra, B., Mèndez, M., Homan, J., \& Belloni, T. M. 2010, MNRAS, submitted

Arnaud, K. A. 1996, in ASP Conf. Ser, 101, Astronomical Data Analysis Software and Systems V, ed. G. H. Jacoby \& J. Barnes (San Francisco, CA: ASP), 17

Ballantyne, D. R. 2004, MNRAS, 351, 57

Ballantyne, D. R., Ross, R. R., \& Fabian, A. C. 2001, MNRAS, 327, 10

Ballantyne, D. R., \& Strohmayer, T. E. 2004, ApJ, 602, L105

Barret, D. 2001, Adv. Space Res., 28, 307

Barret, D., Olive, J. F., Boirin, L., Done, C., Skinner, G. K., \& Grindlay, J. E. 2000, ApJ, 533, 329

Barret, D., Olive, J.-F., \& Miller, M. C. 2005, MNRAS, 361, 855

Barret, D., Olive, J.-F., \& Miller, M. C. 2006, MNRAS, 370, 1140

Barret, D., et al. 2008, Proc. SPIE, 7011, 70110E

Belloni, T., Méndez, M., \& Homan, J. 2005, A\&A, 437, 209

Bhattacharyya, S., \& Strohmayer, T. E. 2007, ApJ, 664, L103

Bhattacharyya, S., Thampan, A. V., Misra, R., \& Datta, B. 2000, ApJ, 542, 473

Boirin, L., Barret, D., Olive, J. F., Bloser, P. F., \& Grindlay, J. E. 2000, A\&A, 361,121

Boirin, L., Méndez, M., Díaz Trigo, M., Parmar, A. N., \& Kaastra, J. S. 2005, A\&A, 436, 195

Boutelier, M., Barret, D., Lin, Y., \& Török, G. 2010, MNRAS, 401, 1290

Boutelier, M., Barret, D., \& Miller, M. C. 2009, MNRAS, 399, 1901

Brandt, W. M., \& Matt, G. 1994, MNRAS, 268, 1051

Brenneman, L. W., \& Reynolds, C. S. 2006, ApJ, 652, 1028

Cackett, E. M., Altamirano, D., Patruno, A., Miller, J. M., Reynolds, M., Linares, M., \& Wijnands, R. 2009a, ApJ, 694, L21

Cackett, E. M., et al. 2008, ApJ, 674, 415

Cackett, E. M., et al. 2009b, ApJ, 690, 1847

Casares, J., Cornelisse, R., Steeghs, D., Charles, P. A., Hynes, R. I., O’Brien, K., \& Strohmayer, T. E. 2006, MNRAS, 373, 1235

Christian, D. J., \& Swank, J. H. 1997, ApJS, 109, 177

D’Aì, A., Iaria, R., Di Salvo, T., Matt, G., \& Robba, N. R. 2009, ApJ, 693, L1

D'Aì, A., et al. 2010, A\&A, 516, A36

Davis, J. E. 2001, ApJ, 562, 575

Day, C. S. R., \& Done, C. 1991, MNRAS, 253, 35P

Di Salvo, T., Iaria, R., Burderi, L., \& Robba, N. R. 2000a, ApJ, 542, 1034 
Di Salvo, T., et al. 2000b, ApJ, 544, L119

Di Salvo, T., et al. 2009c, MNRAS, 398, 2022

Díaz Trigo, M., Parmar, A. N., Boirin, L., Méndez, M., \& Kaastra, J. S. 2006, A\&A, 445, 179

Díaz Trigo, M., Parmar, A. N., Miller, J., Kuulkers, E., \& Caballero-García, M. D. 2007, A\&A, 462, 657

Done, C., Życki, P. T., \& Smith, D. A. 2002, MNRAS, 331, 453

Elebert, P., Callanan, P. J., Torres, M. A. P., \& Garcia, M. R. 2009, MNRAS, 395, 2029

Fabian, A. C., Rees, M. J., Stella, L., \& White, N. E. 1989, MNRAS, 238, 729

Fabian, A. C., et al. 2009, Nature, 459, 540

Farinelli, R., Frontera, F., Zdziarski, A. A., Stella, L., Zhang, S. N., van der Klis, M., Masetti, N., \& Amati, L. 2005, A\&A, 434, 25

Fender, R. P., \& Hendry, M. A. 2000, MNRAS, 317, 1

Galloway, D. K., \& Cumming, A. 2006, ApJ, 652, 559

Galloway, D. K., Muno, M. P., Hartman, J. M., Psaltis, D., \& Chakrabarty, D. 2008, ApJS, 179, 360

George, I. M., \& Fabian, A. C. 1991, MNRAS, 249, 352

Gierliński, M., \& Done, C. 2002, MNRAS, 337, 1373

Gierliński, M., Zdziarski, A. A., Poutanen, J., Coppi, P. S., Ebisawa, K., \& Johnson, W. N. 1999, MNRAS, 309, 496

Gilfanov, M., Revnivtsev, M., \& Molkov, S. 2003, A\&A, 410, 217

Gladstone, J., Done, C., \& Gierliński, M. 2007, MNRAS, 378, 13

Grebenev, S. A., \& Sunyaev, R. A. 2002, Astron. Lett., 28, 150

Hellier, C., \& Mukai, K. 2004, MNRAS, 352, 1037

Homan, J., van der Klis, M., Jonker, P. G., Wijnands, R., Kuulkers, E., Méndez, M., \& Lewin, W. H. G. 2002, ApJ, 568, 878

Horowitz, C. J., \& Piekarewicz, J. 2001, Phys. Rev. Lett., 86, 5647

Iaria, R., D’Aí, A., Di Salvo, T., Robba, N. R., Riggio, A., Papitto, A., \& Burderi, L. 2009, A\&A, 505, 1143

Inogamov, N. A., \& Sunyaev, R. A. 1999, Astron. Lett., 25, 269

Jansen, F., et al. 2001, A\&A, 365, L1

Juett, A. M., Schulz, N. S., \& Chakrabarty, D. 2004, ApJ, 612, 308

Juett, A. M., Schulz, N. S., Chakrabarty, D., \& Gorczyca, T. W. 2006, ApJ, 648, 1066

Kubota, A., Makishima, K., \& Ebisawa, K. 2001, ApJ, 560, L147

Kubota, A., et al. 2007, PASJ, 59, 185

Kuulkers, E., den Hartog, P. R., in't Zand, J. J. M., Verbunt, F. W. M., Harris, W. E., \& Cocchi, M. 2003, A\&A, 399, 663

Kuulkers, E., Parmar, A. N., Owens, A., Oosterbroek, T., \& Lammers, U. 1997, A\&A, 323, L29

Laming, J. M., \& Titarchuk, L. 2004, ApJ, 615, L121

Laor, A. 1991, ApJ, 376, 90

Lattimer, J. M., \& Prakash, M. 2001, ApJ, 550, 426

Laurent, P., \& Titarchuk, L. 2007, ApJ, 656, 1056

Leahy, D. A., Darbro, W., Elsner, R. F., Weisskopf, M. C., Kahn, S., Sutherland, P. G., \& Grindlay, J. E. 1983, ApJ, 266, 160

Lin, D., Remillard, R. A., \& Homan, J. 2007, ApJ, 667, 1073

Magdziarz, P., \& Zdziarski, A. A. 1995, MNRAS, 273, 837

Markoff, S., \& Nowak, M. A. 2004, ApJ, 609, 972

Markoff, S., Nowak, M. A., \& Wilms, J. 2005, ApJ, 635, 1203

Marsh, T. R., Robinson, E. L., \& Wood, J. H. 1994, MNRAS, 266, 137

Méndez, M. 2006, MNRAS, 371, 1925

Miller, J. M. 2007, ARA\&A, 45, 441
Miller, J. M., Homan, J., Steeghs, D., Rupen, M., Hunstead, R. W., Wijnands, R., Charles, P. A., \& Fabian, A. C. 2006a, ApJ, 653, 525

Miller, M. C., Lamb, F. K., \& Cook, G. B. 1998, ApJ, 509, 793

Miller, J. M., Raymond, J., Fabian, A., Steeghs, D., Homan, J., Reynolds, C., van der Klis, M., \& Wijnands, R. 2006b, Nature, 441, 953

Miller, J. M., Raymond, J., Reynolds, C. S., Fabian, A. C., Kallman, T. R., \& Homan, J. 2008a, ApJ, 680, 1359

Miller, J. M., Reynolds, C. S., Fabian, A. C., Miniutti, G., \& Gallo, L. C. 2009, ApJ, 697, 900

Miller, J. M., et al. 2008b, ApJ, 679, L113

Miller, J. M., et al. 2006c, ApJ, 646, 394

Miniutti, G., Fabian, A. C., Goyder, R., \& Lasenby, A. N. 2003, MNRAS, 344 L22

Miniutti, G., et al. 2009, MNRAS, 398, 255

Mitsuda, K., Inoue, H., Nakamura, N., \& Tanaka, Y. 1989, PASJ, 41, 97

Mitsuda, K., et al. 2007, PASJ, 59, 1

Nayakshin, S., \& Kallman, T. R. 2001, ApJ, 546, 406

Orosz, J. A., \& Kuulkers, E. 1999, MNRAS, 305, 132

Pandel, D., Kaaret, P., \& Corbel, S. 2008, ApJ, 688, 1288

Papitto, A., Di Salvo, T., D’Aì, A., Iaria, R., Burderi, L., Riggio, A., Menna, M. T., \& Robba, N. R. 2009, A\&A, 493, L39

Parmar, A. N., Oosterbroek, T., Boirin, L., \& Lumb, D. 2002, A\&A, 386, 910

Piraino, S., Santangelo, A., \& Kaaret, P. 2000, A\&A, 360, L35

Piraino, S., Santangelo, A., \& Kaaret, P. 2002, ApJ, 567, 1091

Popham, R., \& Sunyaev, R. 2001, ApJ, 547, 355

Psaltis, D., et al. 1999, ApJ, 520, 763

Reis, R. C., Fabian, A. C., Ross, R. R., \& Miller, J. M. 2009a, MNRAS, 395, 1257

Reis, R. C., Fabian, A. C., \& Young, A. J. 2009b, MNRAS, 399, L1

Revnivtsev, M. G., \& Gilfanov, M. R. 2006, A\&A, 453, 253

Reynolds, C. S., Nowak, M. A., Markoff, S., Tueller, J., Wilms, J., \& Young, A. J. 2009, ApJ, 691, 1159

Ross, R. R., \& Fabian, A. C. 2007, MNRAS, 381, 1697

Schmoll, S., et al. 2009, ApJ, 703, 2171

Schulz, N. S. 1999, ApJ, 511, 304

Schulz, N. S., Huenemoerder, D. P., Ji, L., Nowak, M., Yao, Y., \& Canizares, C. R. 2009, ApJ, 692, L80

Shakura, N. I., \& Sunyaev, R. A. 1973, A\&A, 24, 337

Shaposhnikov, N., Titarchuk, L., \& Laurent, P. 2009, ApJ, 699, 1223

Sidoli, L., Oosterbroek, T., Parmar, A. N., Lumb, D., \& Erd, C. 2001, A\&A, 379,540

Steeghs, D., \& Casares, J. 2002, ApJ, 568, 273

Stella, L., \& Vietri, M. 1998, ApJ, 492, L59

Sunyaev, R. A., \& Shakura, N. I. 1986, Soviet Astron. Lett., 12, 117

Titarchuk, L., Kazanas, D., \& Becker, P. A. 2003, ApJ, 598, 411

Titarchuk, L., Laurent, P., \& Shaposhnikov, N. 2009, ApJ, 700, 1831

Ueda, Y., Yamaoka, K., \& Remillard, R. 2009, ApJ, 695, 888

van der Klis, M. 2006, in Compact Stellar X-ray sources, ed. W. H. G. Lewin \& M. van der Klis (Cambridge: Cambridge Univ. Press), 39

Vrtilek, S. D., Swank, J. H., \& Kallman, T. R. 1988, ApJ, 326, 186

White, N. E., Stella, L., \& Parmar, A. N. 1988, ApJ, 324, 363

Wijnands, R., et al. 1997, ApJ, 490, L157

Wijnands, R., et al. 1998, ApJ, 493, L87

Wilkinson, T., \& Uttley, P. 2009, MNRAS, 327, 666 